\title{
55. VARIATIONS IN UPPER CRETACEOUS AND CENOZOIC CALCIUM CARBONATE PERCENTAGES, MAUD RISE, WEDDELL SEA, ANTARCTICA ${ }^{1}$
}

\author{
Suzanne B. O'Connell ${ }^{2}$
}

\begin{abstract}
An almost continuous Upper Cretaceous through Pleistocene biogenic sediment section was recovered from two sites on Maud Rise, a volcanic edifice in the Weddell Sea, off eastern Antarctica. Calcium carbonate values were determined for 1100 closely spaced samples using a Coulometrics $\mathrm{CO}_{2}$ Coulometer.

Following a very brief decrease in the percentage of calcium carbonate immediately above the Cretaceous/Tertiary boundary, values remain high $(\sim 70 \%-80 \%)$, throughout most of the Paleocene, with variations primarily attributed to changes in the relative abundance of terrigenous and biogenic components. A small general decrease in calcium carbonate is observed from the upper Paleocene to lower middle Eocene. Eocene values continue to show small to moderate fluctuations. These fluctuations become more pronounced in the Oligocene as biosiliceous and carbonate sediments are mixed and interlayered. A distinct decrease in the calcium carbonate component is observed in the upper Oligocene through lower middle Miocene. Calcium carbonate becomes dominant again in the middle and lower upper Miocene, followed by almost exclusive biosiliceous sedimentation until the Pleistocene, where foraminifer-dominated calcareous ooze was recovered. Interpretation of this data will be carried out when a more finalized chronostratigraphy for the sequence has been produced.
\end{abstract}

\section{INTRODUCTION}

The calcium carbonate content of the sediment column is a function of production, dissolution, and dilution. In modern marine sections collected by piston cores, calcium carbonate stratigraphy commonly parallels stable isotope stratigraphy and has been used for stratigraphic correlation and to interpret global climatic variations (e.g., Hayes et al., 1969; Prell, 1978; Dunn et al., 1981). With the development of the hydraulic piston corer (HPC) and the extended core barrel (XCB), these studies were extended to include the remainder of the Cenozoic (e.g., Gardner, 1982; Pisias and Prell, 1985; Pisias et al., 1985).

During ODP Leg 113, an almost continuous Miocene to Cretaceous calcium carbonate section was recovered at Maud Rise, in the Weddell Sea (Figs. 1 and 2). Closely spaced calcium carbonate analyses were carried out on these samples to monitor percent variations and to relate them to glacial and oceanographic changes. Only the data are presented in this paper because more detailed interpretive studies require an accurate chronostratigraphy, which is still being determined (see Gersonde et al., this volume; Thomas et al., this volume).

\section{SITE LOCATION AND BACKGROUND}

Maud Rise is a volcanic edifice, located in the present-day Antarctic surface water mass (Fig. 1). Two sites were drilled on Maud Rise at different water depths as part of a depth transect to examine the history of vertical water-mass stratification on Upper Cretaceous through Neogene Antarctic sedimentation (Barker, Kennett, et al., 1988).

Site 689 , in $2080 \mathrm{~m}$ of water, lies near the top of Maud Rise and is the shallower of the two sites (Fig. 1). The section penetrated $297.3 \mathrm{~m}$ of almost pure siliceous and calcareous oozes and chalk, with chert layers at the top and bottom of the sequence (Fig. 2A). The sediments range in age from late Campa-

\footnotetext{
${ }^{1}$ Barker, P. F., Kennett, J. P., et al., 1990. Proc. ODP, Sci. Results, 113: College Station, TX (Ocean Drilling Program).

2 Ocean Drilling Program and Department of Oceanography, Texas A\&M University, College Station, TX 77840. Present address: Department of Earth and Environmental Sciences, Wesleyan University, Middletown, CT 06457.
}

nian?/early Maestrichtian to Pliocene. Sedimentary hiatuses or condensed sequences are present throughout the section (Fig. 2A). Three lithologic units were identified: Unit I (0-31.0 mbsf) of middle/late Miocene to Pliocene age is dominated by biosiliceous ooze; Unit II (31.0-149.1 mbsf) consists of a mixture of biosiliceous (predominantly diatom) and calcareous (predominantly nannofossil) oozes ranging in age from late Eocene to middle/late Miocene; Unit III (149.1-297.3 mbsf) is represented by nannofossil ooze and chalk, with varying amounts of foraminifers and ranging in age from late Campanian?/early Maestrichtian to late Eocene (Barker, Kennett, et al., 1988). Hiatuses were identified at the Cretaceous-Tertiary, lower-upper Paleocene; upper Paleocene-middle Eocene; lower-upper Oligocene; lower-middle Miocene; and lower-upper Pliocene boundaries.

At Site 690 , in $2914 \mathrm{~m}$ of water, on the southwestern flank of Maud Rise (Fig. 1), $321.1 \mathrm{~m}$ of sediment was penetrated, ranging in age from Campanian to Pleistocene (Fig. 2B). Sediment in the upper half of the sequence is almost pure siliceous and calcareous ooze with some mixed calcareous ooze/chalk intervals. Terrigenous sediment, dominantly fine grained, was recovered with the biogenic sediments in the lower half of the section. Chert was recovered only in the basal sediments. Compared to Site 689 , fewer hiatuses are present and microfossil preservation is better. A major hiatus spans the middle-upper Eocene and part of the upper Eocene/lower Oligocene. As at Site 689, the upper Oligocene-lower Miocene and lower-middle Miocene hiatuses are present.

Five lithologic units were identified: Unit I, 0-24.2 mbsf, of late Miocene to Pleistocene age, consists of $2.2 \mathrm{~m}$ of foraminiferal ooze underlain by $22.0 \mathrm{~m}$ of biosiliceous ooze; Unit II, 24.2-92.9 mbsf, of early Oligocene to late Miocene age, is represented by a variety of pure and mixed siliceous and calcareous oozes, Unit III, 92.9-137.8 mbsf, of early Eocene to early Oligocene age, is dominated by calcareous ooze. Unit IV, 137.8281.1 mbsf, of late Maestrichtian to early Eocene age, is composed of calcareous ooze/chalk with varying amounts of terrigenous components (clay, quartz, mica). Unit V, 281.1-317.0 mbsf, of late Campanian to early Maestrichtian age, consists of terrigenous components, volcanic glass, and calcium carbonate ooze-chalk. Unit V is directly underlain by basaltic basement. 


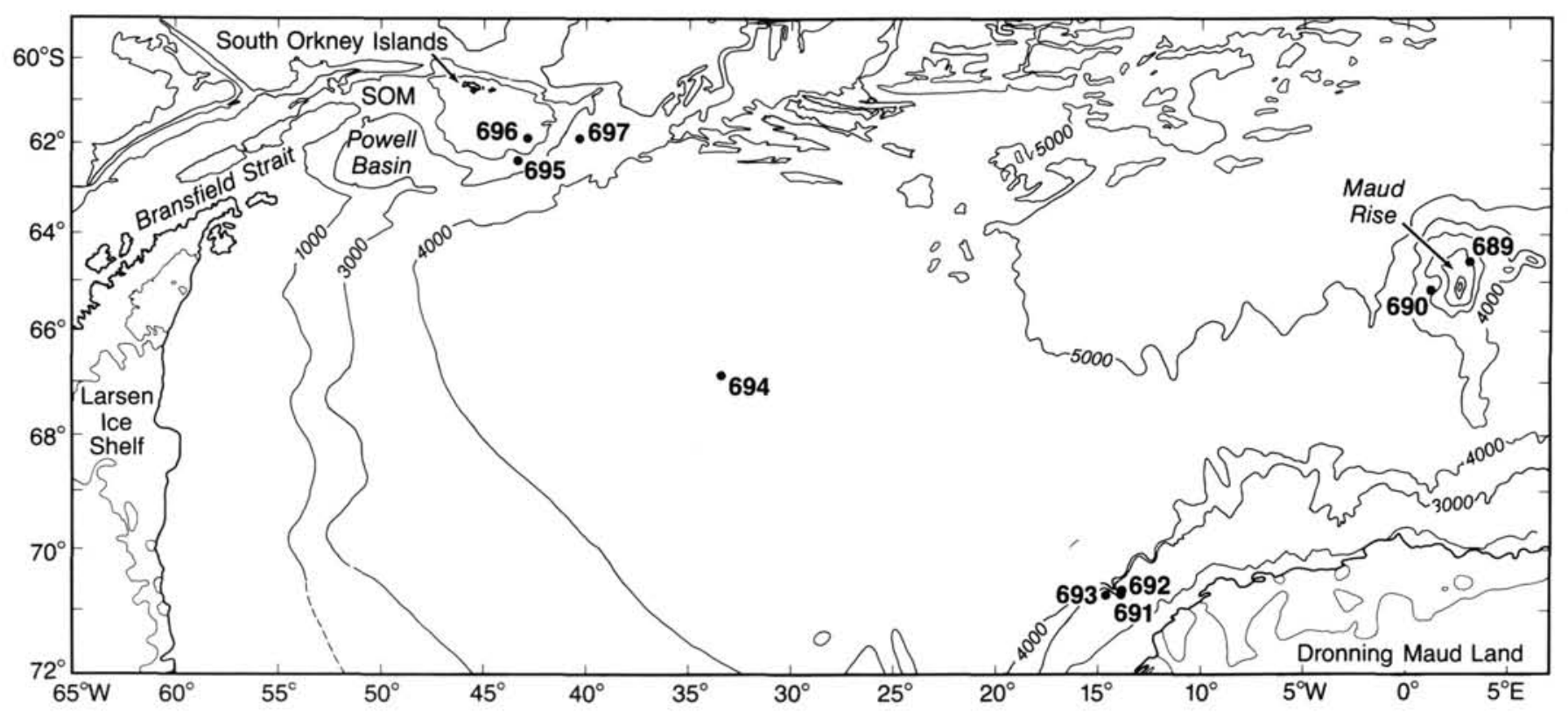

Figure 1. Generalized bathymetry (in meters) and location map, showing Maud Rise in the Weddell Sea and the location of ODP Leg 113 sites (from Barker, Kennett, et al., 1988).

\section{METHODS}

Two $\mathrm{cm}^{3}$ samples were taken every $20-50 \mathrm{~cm}$ throughout the Cenozoic calcium carbonate intervals of Sites 689 and 690 and at wider spacing in siliceous and older intervals. Widely spaced samples within these intervals are due to poor core recovery or poor core quality. Approximately 1100 samples were analyzed. Depths given are from the top of the sampled interval. Samples were dried in either a $100^{\circ} \mathrm{C}$ oven or in a freeze dryer, powdered, weighed to the nearest milligram, and analyzed for calcium carbonate using a Coulometrics $\mathrm{CO}_{2}$ Coulometer. The Coulometer has an accuracy of $0.15 \%( \pm 0.2 \mathrm{mg})$.

The Coulometrics titration technique measures all of the $\mathrm{CO}_{2}$ that is liberated by acidifying and heating sediment samples in a closed system. To do this, the powdered samples are placed in a test tube that is attached to the Coulometer, placed in a heat shield, and $2 \mathrm{~N} \mathrm{HCl}$ is pumped into the test tube. The liberated $\mathrm{CO}_{2}$ is transferred through scrubbers (solutions to remove interfering substances) by a $\mathrm{CO}_{2}$-free gas into an absorption cell where it is titrated through coulometric means. The absorption cell contains an aqueous medium of thanolamine and a coulometric indicator. The interaction of the $\mathrm{CO}_{2}$ and the cell solution creates a titratable acid. The coulometer forms a base electrically and titrates to an end point determined by the optical transmission of the indicator (Huffman, 1977). The pulse output was scaled and fed to a counter in terms of $\mathrm{CO}_{2}$. A stable Coulometer reading indicates that all of the $\mathrm{CO}_{2}$ has been evolved and titrated. The reading was recorded and the results were calculated in micrograms carbon and $\mathrm{CaCO}_{3}\left(\% \mathrm{CaCO}_{3}=\% \mathrm{C}_{\text {inorg }}\right.$ $\times$ 8.334). This method of calculation assumes that all of the $\mathrm{CO}_{2}$ is liberated from calcium carbonate, but some of the $\mathrm{CO}_{2}$ may be liberated from dolomite, siderite, or other carbonate minerals.

Age determinations are from Gersonde et. al. (this volume) and Thomas et al. (this volume), based on biostratigraphic correlations using the Berggren et al. (1985a; 1985b) time scale.

\section{RESULTS}

Calcium carbonate percentages exhibit a wide range of variation in the Maud Rise sediments (0\%-100\%) Tables 1-5; Figs. 3-7).

\section{Upper Cretaceous}

Upper Cretaceous samples range from $65 \%$ to $100 \% \mathrm{CaCO}_{3}$ at Site 689 and $56 \%$ to $92 \%$ at Site 690 , with no systematic trend. Cyclic color variations, which coincide with calcium carbonate variations, are observed in the sediment. These cores were sampled only about every $50 \mathrm{~cm}$ because of the difficulty in distinguishing primary depositional effects from those due to induration and diagenesis. In Hole $690 \mathrm{C}$, relatively high, $(80 \%-$ $90 \%$ ) calcium carbonate values are present below the Cretaceous/Tertiary (K/T) boundary (Table 5, Fig. 7). A sharp, brief drop occurs immediately following the boundary $(48 \%)$, followed by values in the high $80 \%$ 's.

\section{Paleocene}

The amount of Paleocene sediment recovery was very different at the two sites, primarily because of the hiatuses at Site 689. At Site $689,6 \mathrm{~m}$ of lower Paleocene and $25 \mathrm{~m}$ of upper $\mathrm{Pa}$ leocene were recovered (Table 1, Fig. 3). At Site 690, $21 \mathrm{~m}$ of lower Paleocene and $61 \mathrm{~m}$ of upper Paleocene sediment were recovered (Tables 4 and 5; Figs. 6 and 7). Lower Paleocene sediments have calcium carbonate values that average between $87 \%$ and $97 \%$ at Site 689 and $55 \%$ and $100 \%$ at Site 690 . Upper Paleocene sediments also show a narrow range at Site $689(82 \%$ $99 \%)$ and a wide range at Site $690(56 \%-99 \%)$. At Site 690 , the calcium carbonate percentages exhibit an overall small decreasing trend superimposed on these variations. The lower values are associated with darker, more clay rich layers, although there is not always a direct correlation with darkness and lower calcium carbonate values (Fig. 8). The clay minerals are dominantly composed of smectite (Robert and Maillot, this volume), and no mineralogical change is associated with the fluctuations in calcium carbonate values.

Recovery of lower Eocene sediments was much better at Site 690 than at Site 689, but recovery in the remainder of the Eocene was better at Site 689 (Tables 1 and 4; Figs. 3 and 6). The trend of decreasing calcium carbonate continues in the lower Eocene (Site 690), with values ranging between $58 \%$ and $91 \%$. A small overall increase is observed in the remainder of the Eocene with values ranging from $70 \%$ to $100 \%$ (Site 689), and $64 \%$ to $90 \%$ (Site 690 ) for the middle Eocene, and $77 \%$ to $98 \%$ 

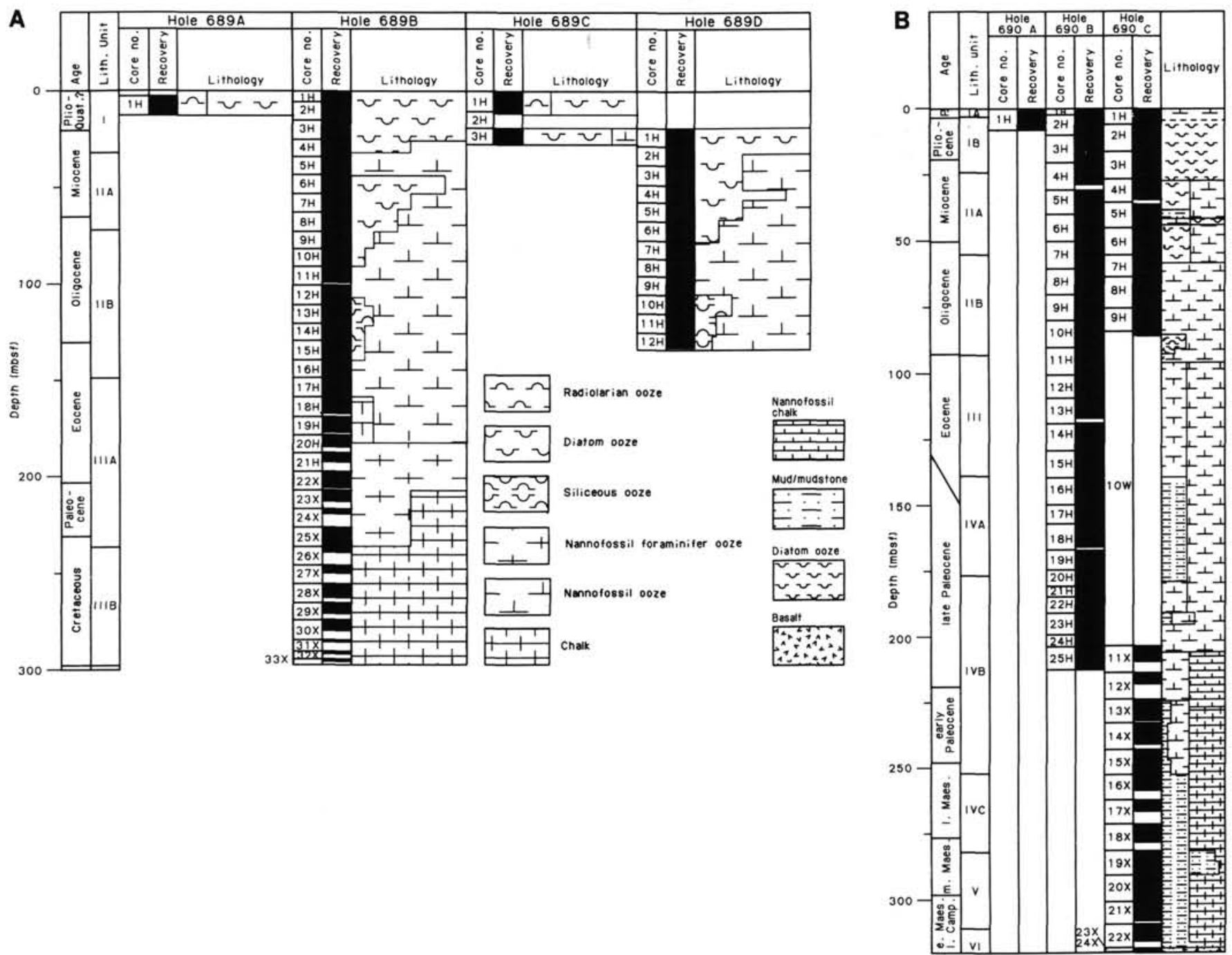

Figure 2. A. Simplified lithology for Site 689, including core number and recovery; ages are from shipboard data. B. Simplified lithology for Site 690 , including core number and recovery; ages are from shipboard data (from Barker, Kennett, et al., 1988).

(Site 689) and 62\% to $96 \%$ (Site 690) for the upper Eocene. Eocene sediments at both sites are primarily white nannofossil ooze, and the minor clay component is dominated by smectite with an increase in illite in the upper Eocene (Robert and Maillot, this volume).

The Oligocene section at Site 690 is two-thirds as thick as at Site $689,40 \mathrm{~m}$ vs. $58 \mathrm{~m}$ (Tables 1 and 4; Figs. 3 and 6). Calcium carbonate values show the following ranges: lower Oligocene, $60 \%-100 \%$ (Site 689) and 51\%-85\% (Site 690), and upper Oligocene, 60\%-99\% (Site 689), and 39\%-93\% (Site 690). At both sites the range of calcium carbonate percentages becomes more variable upsection, with a distinct decrease in overall abundance in the upper Oligocene. Unlike the variations in the lower Paleocene, which were attributable to the influx of terrigenous sediment, the Oligocene fluctuations are attributed to variations in the siliceous component. Although these variations are not as readily distinguishable by color, they are commonly associated with textural changes (Fig. 9). There is also a change in clay mineralogy: smectite is replaced by illite associated with chlorite and mixed layer clays. This change is attributed to a change in weathering conditions on Antarctica caused by the development of cold and/or dry climate. The clay mineral change is observed throughout the entire Oligocene (Robert and Maillot, this volume).

\section{Neogene}

A hiatus marks the Oligocene-Miocene (Fig. 9) and earlymiddle Miocene boundary at both sites. The most marked change and highest variability in calcium carbonate values occurs during the Miocene (Tables 1-4; Figs. 3-6) with values as follows; lower Miocene, 0\%-82\% (Site 689) and 37\%-70\% (Site 690); middle Miocene, 0\%-91\% (Site 689) and 0\%-87\% (Site 690); and upper Miocene, 0\%-46\% (Site 689) and 0\%-83\% (Site 690). Throughout the lower and lower middle Miocene there is a gradual, although highly variable, decrease in calcium carbonate values. At both sites, the middle middle Miocene is a biosiliceous interval almost completely devoid of carbonate. The siliceous-rich interval is overlain by a carbonate-rich interval that stops at the middle-late Miocene boundary at Site 689 and continues into the upper Miocene at Site 690. Although terrigenous material is sparse, smectite is again thedominant clay mineral (Robert and Maillot, this volume).

During the Pliocene the sediments consist of almost exclusive biosiliceous sediments (Tables 1-4; Figs. 3-6). This domi- 
Table 1. Calcium carbonate percentages for Hole 689B, taken between 4.00 and 291.50 mbsf depths. Data plotted in Figure 3.

\begin{tabular}{|c|c|c|c|c|c|c|c|c|c|c|c|c|c|c|c|c|c|c|c|c|}
\hline $\begin{array}{l}\text { Hole, } \\
\text { interv }\end{array}$ & core, & & $\begin{array}{l}\text { Depth } \\
\text { (mbsf) }\end{array}$ & $\begin{array}{c}\mathrm{CaCO} 3 \\
(\%)\end{array}$ & $\begin{array}{l}\text { Hole, } \\
\text { interv }\end{array}$ & $\begin{array}{l}\text { core, s } \\
\text { lal (cr }\end{array}$ & & $\begin{array}{c}\text { Depth } \\
\text { (mbsf) }\end{array}$ & $\begin{array}{c}\mathrm{CaCO}_{3} \\
(\%)\end{array}$ & $\begin{array}{l}\text { Hole, } \\
\text { interv }\end{array}$ & $\begin{array}{l}\text { core, } \\
\text { al ( }\end{array}$ & $\begin{array}{l}\text { e, se } \\
\text { (cm }\end{array}$ & & $\begin{array}{l}\text { Depth } \\
\text { (mbsf) }\end{array}$ & $\begin{array}{c}\mathrm{CaCO} 3 \\
(\%)\end{array}$ & $\begin{array}{l}\text { Hole, c } \\
\text { interva }\end{array}$ & $\begin{array}{l}\text { core, s } \\
\text { al (cr }\end{array}$ & & & $\begin{array}{l}\text { Depth } \\
\text { (mbsf) }\end{array}$ \\
\hline $689 B$ & 10 & 101 & 04.00 & 00.00 & $689 B$ & 903 & 119 & 76.29 & 84.04 & $689 B$ & 130 & 01 & 63 & 111.23 & 63.90 & $689 \mathrm{~B}$ & 1603 & 036 & 62 & 143.02 \\
\hline $689 \mathrm{~B}$ & 201 & 23 & 05.53 & 00.60 & $689 B$ & 904 & 22 & 76.82 & 88.71 & $689 B$ & 130 & 01 & 69 & 111.29 & 70.17 & $689 B$ & 1603 & 03 & 73 & 143.13 \\
\hline $689 B$ & 203 & 20 & 08.50 & 02.90 & $689 \mathrm{~B}$ & 904 & 70 & 77.30 & 92.37 & $689 B$ & 130 & 01 & 120 & 111.80 & 69.68 & $689 \mathrm{~B}$ & 160 & 031 & 118 & 143.58 \\
\hline $689 \mathrm{~B}$ & 204 & 145 & 11.25 & 00.30 & $689 \mathrm{~B}$ & 904 & 90 & 77.50 & 85.10 & $689 \mathrm{~B}$ & 130 & 01 & 134 & 111.94 & 78.63 & $689 \mathrm{~B}$ & 160 & 042 & 23 & 144.13 \\
\hline $689 B$ & 205 & 20 & 11.50 & 01.20 & $689 \mathrm{~B}$ & 904 & 114 & 77.74 & 85.72 & $689 \mathrm{~B}$ & 130 & 02 & 22 & 112.32 & 86.72 & $689 \mathrm{~B}$ & 160 & 047 & 73 & 144.63 \\
\hline $689 B$ & 301 & 20 & 15.00 & 00.30 & $689 B$ & 904 & 117 & 77.77 & 89.20 & $689 \mathrm{~B}$ & 130 & 02 & 69 & 112.79 & 78.82 & $689 \mathrm{~B}$ & 160 & 041 & 118 & 145.08 \\
\hline $689 B$ & 303 & 20 & 18.00 & 00.20 & $689 B$ & 905 & 22 & 78.32 & 91.30 & $689 B$ & 13 & 02 & 122 & 113.32 & 69.80 & $689 \mathrm{~B}$ & 1605 & 052 & 23 & 145.63 \\
\hline $689 B$ & 304 & 120 & 20.50 & 00.30 & $689 B$ & 905 & 70 & 78.80 & 98.29 & $689 B$ & 130 & 03 & 22 & 113.82 & 83.25 & $689 B$ & 1605 & 05 & 63 & 146.03 \\
\hline $689 \mathrm{~B}$ & 305 & 21 & 21.00 & 01.00 & $689 B$ & 905 & 119 & 79.29 & 87.88 & $689 \mathrm{~B}$ & 130 & 03 & 63 & 114.23 & 90.00 & $689 B$ & 1605 & 057 & 73 & 146.13 \\
\hline $689 B$ & 401 & 63 & 24.93 & 03.90 & $689 \mathrm{~B}$ & 906 & 8 & 79.68 & 75.50 & $689 \mathrm{~B}$ & 130 & 03 & 69 & 114.29 & 88.29 & $689 B$ & 160 & 051 & 118 & 146.58 \\
\hline $689 B$ & 403 & 63 & 27.93 & 00.50 & $689 \mathrm{~B}$ & 906 & 22 & 79.82 & 81.70 & $689 \mathrm{~B}$ & 13 & 03 & 122 & 114.82 & 89.21 & $689 B$ & 1606 & 062 & 23 & 147.13 \\
\hline $689 B$ & 404 & 145 & 30.25 & 00.20 & $689 B$ & 906 & 70 & 80.30 & 89.20 & $689 \mathrm{~B}$ & 130 & 04 & 22 & 115.32 & 79.13 & $689 B$ & 1606 & 067 & 73 & 147.63 \\
\hline $689 B$ & 405 & 63 & 30.93 & 50.70 & $689 \mathrm{~B}$ & $9 \propto c$ & 10 & 80.55 & 82.88 & $689 \mathrm{~B}$ & 130 & 04 & 69 & 115.79 & 89.71 & $689 B$ & 160 & 061 & 118 & 148.08 \\
\hline $689 \mathrm{~B}$ & 405 & 92 & 31.22 & 62.10 & $689 B$ & 1001 & 22 & 81.92 & 63.47 & $689 \mathrm{~B}$ & 13 & 04 & 122 & 116.32 & 91.40 . & $689 B$ & 160 & 072 & 23 & 148.63 \\
\hline 6898 & 406 & 90 & 32.70 & 81.30 & $689 B$ & 1001 & 68 & 82.38 & 80.80 & $689 B$ & 130 & 05 & 22 & 116.82 & 89.29 & 6898 & $16 \mathrm{cc}$ & $c$ & 9 & 149.01 \\
\hline 6898 & 502 & 90 & 36.20 & 81.30 & $689 \mathrm{~B}$ & 1001 & 123 & 82.93 & 82.53 & $689 B$ & 130 & 05 & 63 & 117.23 & 92.60 & $689 \mathrm{~B}$ & 170 & 01 & 22 & 149.32 \\
\hline 6898 & 504 & 90 & 39.20 & 91.30 & $689 \mathrm{~B}$ & 1002 & 22 & 83.42 & 79.80 & $689 B$ & 13 & 05 & 120 & 117.80 & 84.30 & $689 B$ & 170 & 01 & 59 & 149.69 \\
\hline 6898 & 504 & 145 & 39.75 & 89.00 & $689 B$ & 1002 & 68 & 83.88 & 76.96 & $689 \mathrm{~B}$ & 130 & 06 & 22 & 118.32 & 85.12 & $689 B$ & 170 & 01 & 73 & 149.83 \\
\hline $689 B$ & 506 & 90 & 42.20 & 90.00 & $689 \mathrm{~B}$ & 1002 & 90 & 84.10 & 98.50 & 6898 & 130 & 06 & 71 & 118.81 & 84.86 & 6898 & 170 & 011 & 127 & 150.37 \\
\hline $689 B$ & 602 & 90 & 45.70 & 07.80 & $689 B$ & 1002 & 123 & 84.43 & 86.96 & $689 B$ & 13 & 06 & 125 & 119.35 & 72.81 & 6898 & 1702 & 022 & 22 & 150.82 \\
\hline $689 \mathrm{~B}$ & 604 & 67 & 48.47 & 01.20 & $689 \mathrm{~B}$ & 1003 & 22 & 84.92 & 84.46 & 6898 & 130 & 07 & 22 & 119.82 & 78.89 & $689 B$ & 170 & 021 & 127 & 151.87 \\
\hline $689 \mathrm{~B}$ & 604 & 120 & 49.00 & 00.80 & $689 \mathrm{~B}$ & 1003 & 68 & 85.38 & 86.38 & $689 B$ & $13 c$ & $c$ & 21 & 120.13 & 82.55 & $689 B$ & 1703 & 032 & 22 & 152.32 \\
\hline $689 B$ & 606 & 90 & 51.70 & 36.80 & $689 \mathrm{~B}$ & 1003 & 123 & 85.93 & $\begin{array}{l}88.30 \\
88.88\end{array}$ & $689 B$ & 140 & 01 & 23 & 120.43 & 85.51 & $689 B$ & 1703 & 03 & 58 & 152.68 \\
\hline $689 B$ & 701 & 15 & 53.05 & 08.41 & $689 \mathrm{~B}$ & 1004 & 22 & 86.42 & $\begin{array}{l}00.00 \\
91.21\end{array}$ & $689 B$ & 140 & 01 & 62 & 120.82 & 85.10 & $689 B$ & 1703 & 03 & 73 & 152.83 \\
\hline $689 B$ & 701 & 60 & 53.50 & 26.74 & $689 B$ & 1004 & 68 & 86.88 & 79.80 & $689 B$ & 140 & 01 & 70 & 120.90 & 81.05 & $689 B$ & 170 & 031 & 127 & 153.37 \\
\hline $689 B$ & 701 & 110 & 54.00 & 61.39 & $689 B$ & 1004 & 90 & 87.10 & 84.10 & $689 B$ & 14 & 01 & 120 & 121.40 & 81.59 & $689 \mathrm{~B}$ & 170 & 042 & 22 & 153.82 \\
\hline $689 \mathrm{~B}$ & 702 & 15 & 54.55 & 19.99 & $689 B$ & 1004 & 123 & 87.43 & 89.55 & $689 B$ & 140 & 02 & 23 & 121.93 & 93.80 & $689 B$ & 170 & 04 & 73 & 154.33 \\
\hline $689 B$ & 702 & 60 & 55.00 & 25.65 & $689 \mathrm{~B}$ & 1005 & 22 & 87.92 & 83.71 & 6898 & 140 & 02 & 70 & 122.40 & 89. & $689 B$ & 1705 & 05 & 22 & 155.32 \\
\hline $689 B$ & 702 & 90 & 55.30 & 54.60 & $689 \mathrm{~B}$ & 1005 & 68 & 88.38 & 83.55 & $689 \mathrm{~B}$ & 140 & 02 & 123 & 122.93 & 87.76 & $689 B$ & 170 & 05 & 58 & 155.68 \\
\hline $689 \mathrm{~B}$ & 702 & 110 & 55.50 & 25.91 & $689 B$ & 1005 & 123 & 88.93 & 89.21 & $689 B$ & 140 & 03 & 23 & 123.43 & 91.55 & 6898 & 170 & 057 & 73 & 155.83 \\
\hline $689 B$ & 703 & 15 & 56.05 & 00.83 & $689 \mathrm{~B}$ & 1006 & 22 & 89.42 & 79.38 & $689 \mathrm{~B}$ & 140 & 03 & 63 & 123.83 & 87.40 & $689 B$ & 170 & 0512 & 127 & 156.37 \\
\hline $689 B$ & 703 & 60 & 56.50 & 04.00 & $689 B$ & 1006 & 68 & 89.88 & 83.38 & $689 \mathrm{~B}$ & 140 & 03 & 70 & 123.90 & 84.90 & $689 B$ & 1706 & 062 & 22 & 156.82 \\
\hline $689 B$ & 703 & 110 & 57.00 & 11.50 & $689 B$ & 1006 & 90 & 90.10 & 76.90 & $689 \mathrm{~B}$ & 14 & 03 & 123 & 124.43 & 86.93 & $689 B$ & 1707 & 07 & 22 & 158.32 \\
\hline $689 B$ & 704 & 15 & 57.55 & 03.50 & $689 \mathrm{~B}$ & 1101 & 45 & 91.75 & 79.38 & $689 B$ & 140 & 04 & 23 & 124.93 & 90.63 & $689 B$ & $17 c c$ & $x$ & 9 & 158.79 \\
\hline $689 B$ & 704 & 60 & 58.00 & 40.90 & $689 B$ & 1101 & 73 & 92.03 & 84.88 & $689 \mathrm{~B}$ & 140 & 04 & 70 & 125.40 & 87.11 & $689 B$ & 180 & 01 & 22 & 159.02 \\
\hline 6898 & 704 & 90 & 58.30 & 37.70 & $689 B$ & 1101 & 90 & 92.20 & 83.10 & $689 \mathrm{~B}$ & 14 & 04 & 123 & 125.93 & 89.50 & $689 B$ & 180 & 017 & 74 & 159.54 \\
\hline $689 B$ & 704 & 110 & 58.50 & 23.57 & $689 B$ & 1101 & 125 & 92.55 & 79.13 & $689 \mathrm{~B}$ & 140 & 05 & 23 & 126.43 & 84.85 & $689 B$ & 180 & 0112 & 123 & 160.03 \\
\hline $689 B$ & 705 & 15 & 59.05 & 57.98 & $689 \mathrm{~B}$ & 1102 & 23 & 93.03 & 78.88 & $689 B$ & 140 & 05 & 61 & 126.81 & 87.10 & $689 \mathrm{~B}$ & 1802 & 222 & 22 & 160.52 \\
\hline 6898 & 705 & 60 & 59.50 & 81.55 & $689 B$ & 1102 & 73 & 93.53 & 86.13 & $689 B$ & 140 & 05 & 70 & 126.90 & 86.62 & $689 B$ & 1802 & 02 & 74 & 161.04 \\
\hline $689 \mathrm{~B}$ & 705 & 110 & 60.00 & 73.80 & $689 \mathrm{~B}$ & 1102 & 125 & 94.05 & 82.21 & $689 B$ & 14 & 05 & 120 & 127.40 & 87.95 & $689 B$ & 1802 & 029 & 90 & 161.20 \\
\hline $689 B$ & 706 & 15 & 60.55 & 57.14 & $689 \mathrm{~B}$ & 1103 & 23 & 94.53 & 90.04 & $689 B$ & 140 & 06 & 23 & 127.93 & 87.22 & $689 \mathrm{~B}$ & 180 & 021 & 123 & 161.53 \\
\hline 6898 & 706 & 60 & 61.00 & 40.56 & $689 \mathrm{~B}$ & 1103 & 73 & 95.03 & 91,54 & $689 B$ & 140 & 06 & 70 & 128.40 & 88.38 & $689 B$ & 1803 & 032 & 22 & 162.02 \\
\hline 6898 & 706 & 90 & 61.30 & 00.20 & $689 \mathrm{~B}$ & 1103 & 90 & 95.20 & 79.10 & $689 \mathrm{~B}$ & 14 & 06 & 123 & 128.93 & 77.38 & $689 B$ & 1803 & 037 & 74 & 162.54 \\
\hline $689 \mathrm{~B}$ & 706 & 110 & 61.50 & 40.48 & $689 B$ & 1103 & 125 & 95.55 & 87.21 & $689 B$ & 140 & 07 & 25 & 129.45 & 82.05 & $689 B$ & 180 & 031 & 123 & 163.03 \\
\hline $689 B$ & 707 & 15 & 62.05 & 24.07 & $689 \mathrm{~B}$ & 1104 & 23 & 96.03 & 81.80 & $689 B$ & $14 \mathrm{c}$ & $c c$ & 9 & 129.61 & 85.29 & $689 B$ & 180 & 042 & 22 & 163.52 \\
\hline $689 B$ & $7 c c$ & 5 & 62.29 & 13.99 & $689 \mathrm{~B}$ & 1104 & 73 & 96.53 & 72.47 & $689 B$ & 150 & 01 & 22 & 130.12 & 82.84 & $689 B$ & 180 & 04 & 74 & 164.04 \\
\hline $689 \mathrm{~B}$ & 801 & 17 & 62.67 & 68.47 & $689 \mathrm{~B}$ & 1104 & 125 & 97.05 & 89.13 & $689 B$ & 150 & 01 & 72 & 130.62 & 84.43 & $689 B$ & 180 & 049 & 90 & 164.20 \\
\hline $689 B$ & 801 & 70 & 63.20 & 58.39 & $689 B$ & 1105 & 23 & 97.53 & 81.71 & $689 B$ & 150 & 01 & 64 & 130.64 & 87.50 & $689 \mathrm{~B}$ & 180 & 041 & 112 & 164.42 \\
\hline $689 B$ & 801 & 90 & 63.40 & 51.20 & $689 B$ & 1105 & 73 & 98.03 & 97.29 & $689 B$ & 150 & 01 & 123 & 131.13 & 89.68 & $689 \mathrm{~B}$ & 180 & 041 & 113 & 164.43 \\
\hline $689 B$ & 801 & 118 & 63.68 & 60.89 & $689 \mathrm{~B}$ & 1105 & 90 & 98.20 & 84.90 & 6898 & 150 & 02 & 22 & 131.62 & 88.10 & $689 \mathrm{~B}$ & 180 & 052 & 22 & 165.02 \\
\hline $689 B$ & 802 & 17 & 64.17 & 74.63 & $689 \mathrm{~B}$ & 1105 & 125 & 98.55 & 91.54 & $689 B$ & 150 & 02 & 72 & 132.12 & 91.88 & $689 \mathrm{~B}$ & 180 & 05 & 74 & 165.54 \\
\hline $689 B$ & 802 & 70 & 64.70 & 72.97 & 689 & $11 c c$ & 10 & 98.82 & 81.05 & $689 B$ & 150 & 02 & 123 & 132.63 & 90.49 & $689 \mathrm{~B}$ & 180 & 051 & 123 & 166.03 \\
\hline $689 B$ & 802 & 118 & 65.18 & 60.56 & 689 & 1201 & 21 & 101.21 & 59.89 & $689 B$ & 150 & 03 & 22 & 133.12 & 86.35 & $689 B$ & 180 & 162 & 22 & 166.52 \\
\hline $689 B$ & 803 & 17 & 65.67 & 47.56 & $689 \mathrm{~B}$ & 1201 & 63 & 101.63 & 61.80 & $689 B$ & 150 & 03 & 64 & 133.54 & 89.20 & $689 B$ & $18 \mathrm{CC}$ & $\mathrm{C}$ & 10 & 166.78 \\
\hline $689 B$ & 803 & 70 & 66.20 & 58.14 & 6898 & 1201 & 70 & 101.70 & 70.94 & $689 B$ & 150 & 03 & 72 & 133.62 & 85.55 & $689 \mathrm{~B}$ & 180 & 06 & 90 & 167.20 \\
\hline $689 B$ & 803 & 90 & 66.40 & 63.10 & $689 \mathrm{~B}$ & 1201 & 116 & 102.16 & 67.80 & $689 B$ & 150 & 03 & 123 & 134.13 & 87.08 & $689 B$ & 190 & 01 & 21 & 168.71 \\
\hline $689 B$ & 803 & 118 & 66.68 & 79.97 & 6898 & 1202 & 26 & 102.76 & 65.72 & $689 \mathrm{~B}$ & 150 & 04 & 22 & 134.62 & 88.14 & $689 B$ & 190 & 017 & 73 & 169.23 \\
\hline $689 B$ & 804 & 17 & 67.17 & 74.72 & 6898 & 1202 & 70 & 103.20 & 83.23 & $689 \mathrm{~B}$ & 150 & 04 & 72 & 135.12 & 93.37 & $689 B$ & 190 & 011 & 123 & 169.73 \\
\hline $689 B$ & 804 & 70 & 67.70 & 72.80 & 6898 & 1202 & 118 & 103.68 & 77.64 & $689 B$ & 150 & 04 & 112 & 135.52 & 87.90 & $689 B$ & 1902 & 022 & 21 & 170.21 \\
\hline $689 B$ & 804 & 118 & 68.18 & 70.05 & $689 B$ & 1203 & 21 & 104.21 & 82.71 & $689 B$ & 150 & 041 & 114 & 135.54 & 90.21 & $689 B$ & 1902 & 299 & 90 & 170.90 \\
\hline $689 B$ & 805 & 17 & 68.67 & 73.47 & $689 \mathrm{~B}$ & 1203 & 62 & 104.62 & 85.10 & $689 B$ & 150 & 05 & 22 & 136.12 & 94.55 & $689 B$ & 190 & 021 & 123 & 171.23 \\
\hline 6898 & 805 & 70 & 69.20 & 78.64 & $689 B$ & 1203 & 70 & 104.70 & 60.72 & $689 B$ & 150 & 05 & 64 & 136.54 & 88.80 & $689 B$ & 1903 & 032 & 21 & 171.71 \\
\hline 6898 & 805 & 90 & 69.40 & 68.40 & $689 B$ & 1204 & 112 & 105.10 & 86.00 & $689 \mathrm{~B}$ & 150 & 05 & 72 & 136.62 & 88.63 & $689 B$ & 190 & 031 & 123 & 172.73 \\
\hline $689 B$ & 805 & 118 & 69.68 & 82.55 & $689 \mathrm{~B}$ & 1203 & 116 & 105.16 & 83.71 & $689 B$ & 150 & 05 & 123 & 137.13 & 97.71 & $689 B$ & 190 & 042 & 21 & 173.21 \\
\hline $689 B$ & 806 & 17 & 70.17 & 85.46 & $689 B$ & 1204 & 21 & 105.71 & 88.30 & $689 B$ & 150 & 06 & 22 & 137.62 & 90.06 & $689 \mathrm{~B}$ & 190 & 049 & 90 & 173.90 \\
\hline $689 B$ & 806 & 70 & 70.70 & 75.80 & $689 \mathrm{~B}$ & 1204 & 70 & 106.20 & 97.62 & $689 \mathrm{~B}$ & $15 \mathrm{C}$ & $\mathrm{C} 6$ & 72 & 138.12 & 94.38 & $689 B$ & 190 & 041 & 123 & 174.23 \\
\hline $689 B$ & 806 & 118 & 71.18 & 95.96 & $689 \mathrm{~B}$ & 1204 & 107 & 106.57 & 87.21 & $689 B$ & 150 & 061 & 123 & 138.63 & 94.15 & $689 B$ & 1905 & 552 & 21 & 174.71 \\
\hline $689 B$ & 807 & 10 & 71.60 & 80.88 & $689 \mathrm{~B}$ & 1205 & 21 & 107.21 & 81.99 & $689 B$ & 150 & 07 & 21 & 139.11 & 95.38 & $689 \mathrm{~B}$ & 190 & 051 & 123 & 175.73 \\
\hline $689 B$ & 901 & 22 & 72.32 & 60.14 & $689 \mathrm{~B}$ & 1205 & 64 & 107.64 & 83.00 & $689 \mathrm{~B}$ & 160 & 01 & 23 & 139.63 & 87.02 & $689 B$ & 1906 & 662 & 21 & 176.21 \\
\hline $689 B$ & 901 & 70 & 72.80 & 75.80 & $689 B$ & 1205 & 70 & 107.70 & 85.38 & $689 B$ & $15 c$ & $\infty \quad 1$ & 14 & 139.64 & 83.41 & $689 B$ & 1906 & 6 & 52 & 176.52 \\
\hline $689 B$ & 901 & 119 & 73.29 & 91.05 & $689 B$ & 1205 & 116 & 108.16 & 79.38 & $689 B$ & 160 & 01 & 62 & 140.00 & 95.80 & 6898 & 1906 & 66 & 73 & 176.73 \\
\hline $689 B$ & 902 & 22 & 73.82 & 95.13 & $689 \mathrm{~B}$ & 1206 & 21 & 108.71 & 63.26 & $689 B$ & 160 & 01 & 73 & 140.13 & 94.62 & $689 B$ & 2001 & 1 & 26 & 178.36 \\
\hline $689 B$ & 902 & 70 & 74.30 & 93.38 & $689 \mathrm{~B}$ & 1206 & 70 & 109.20 & 100.00 & $689 \mathrm{~B}$ & 160 & 011 & 118 & 140.58 & 94.55 & $689 \mathrm{~B}$ & 2001 & 18 & 85 & 178.95 \\
\hline $689 B$ & 902 & 90 & 74.50 & 89.60 & $689 \mathrm{~B}$ & 1206 & 116 & 109.66 & 71.13 & 6898 & 160 & 02 & 23 & 141.13 & 96.82 & $689 B$ & 200 & 011 & 125 & 179.35 \\
\hline $689 \mathrm{~B}$ & 902 & 119 & 74.79 & 93.12 & $689 \mathrm{~B}$ & 1207 & 21 & 110.21 & 72.59 & $689 \mathrm{~B}$ & 160 & 02 & 73 & 141.63 & 93.63 & $689 \mathrm{~B}$ & 2002 & 22 & 26 & 179.86 \\
\hline $689 B$ & 903 & 22 & 75.32 & 84.22 & 6898 & $12 \mathrm{cc}$ & 13 & 110.51 & 67.38 & $689 B$ & 160 & 021 & 118 & 142.08 & 93.20 & $689 \mathrm{~B}$ & 2002 & & 85 & 180.45 \\
\hline $689 B$ & 903 & 70 & 75.80 & 97.04 & $689 \mathrm{~B}$ & 1301 & 22 & 110.82 & 75.13 & $689 \mathrm{~B}$ & 160 & 03 & 23 & 142.63 & 93.72 & $689 \mathrm{~B}$ & 2002 & & 90 & 180.50 \\
\hline
\end{tabular}


Table 1 (continued).

\begin{tabular}{|c|c|c|c|c|c|c|c|c|}
\hline $\begin{array}{l}\text { Hole, core } \\
\text { interval }\end{array}$ & & & $\begin{array}{l}\text { Depth } \\
\text { (mbsf) }\end{array}$ & $\begin{array}{c}\mathrm{CaCO} 3 \\
(\%)\end{array}$ & $\begin{array}{l}\text { Hole, core, se } \\
\text { interval (cm }\end{array}$ & & $\begin{array}{l}\text { Depth } \\
\text { (mbsf) }\end{array}$ & $\begin{array}{c}\mathrm{CaCO} 3 \\
(\%)\end{array}$ \\
\hline $689 B \quad 20$ & 02 & 125 & 180.85 & 88.13 & 689B 2701 & 62 & 246.62 & 72.80 \\
\hline $689 B 20$ & 03 & 26 & 181.36 & 84.21 & 68982702 & 21 & 247.71 & 99.71 \\
\hline $689 \mathrm{~B} 20$ & 03 & 85 & 181.95 & 88.54 & 689B 2702 & 120 & 248.70 & 88.38 \\
\hline $689 \mathrm{~B} 20$ & 03 & 125 & 182.35 & 87.54 & $689 B 2703$ & 20 & 249.20 & 89.88 \\
\hline $689 B 20$ & 04 & 26 & 182.86 & 76.13 & $689 B 2703$ & 80 & 249.80 & 78.88 \\
\hline 689820 & 04 & 85 & 183.45 & 82.21 & $689 \mathrm{~B} 27 \mathrm{CC}$ & 4 & 250.11 & 96.21 \\
\hline $689 B 20$ & 04 & 90 & 183.50 & 89.50 & 689B 2801 & 20 & 255.80 & 92.87 \\
\hline $689 B 20$ & 04 & 125 & 183.85 & 83.38 & 689B 2801 & 72 & 256.32 & 85.79 \\
\hline $689 B 20$ & 05 & 26 & 184.36 & 84.29 & $689 \mathrm{~B} 2801$ & 114 & 256.74 & 89.04 \\
\hline $689 B 21$ & 01 & 21 & 188.01 & 90.46 & 689B 2801 & 120 & 256.80 & 86.79 \\
\hline $689 B 21$ & 01 & 72 & 188.52 & 82.38 & $689 \mathrm{~B} 2802$ & 21 & 257.31 & 75.38 \\
\hline $689 B 21$ & 01 & 90 & 188.70 & 93.30 & 689B 2802 & 75 & 257.85 & 86.29 \\
\hline $689 B 21$ & 01 & 127 & 189.07 & 88.21 & $689 B 2802$ & 129 & 258.39 & 90.63 \\
\hline $689 B 21$ & 02 & 21 & 189.51 & 88.38 & $689 B 2803$ & 70 & 259.30 & 85.38 \\
\hline $689 B 21$ & 02 & 72 & 190.02 & 83.71 & $689 B 2803$ & 112 & 259.72 & 91.50 \\
\hline 689821 & 02 & 127 & 190.57 & 82.30 & $689 \mathrm{~B} \quad 28 \quad 03$ & 113 & 259.73 & 83.38 \\
\hline $689 B 21$ & 03 & 21 & 191.01 & 90.29 & $689 B 2804$ & 14 & 260.24 & 73.05 \\
\hline $689 B 21$ & 03 & 22 & 191.02 & 86.46 & $689 B 2804$ & 65 & 260.75 & 78.63 \\
\hline $689 B 21$ & 03 & 72 & 191.52 & 90.38 & $689 B \quad 2804$ & 148 & 261.58 & 77.80 \\
\hline 689821 & 03 & 90 & 191.70 & 94.40 & $689 \mathrm{~B} 28 \mathrm{CC}$ & 16 & 261.76 & 91.13 \\
\hline $689 B 21$ & 03 & 127 & 192.07 & 87.54 & $689 B 28 \mathrm{CC}$ & 22 & 261.82 & 91.71 \\
\hline $689 \mathrm{~B} 21$ & $\mathrm{CC}$ & 9 & 192.17 & 89.21 & $689 B 2901$ & 18 & 265.38 & 87.04 \\
\hline $689 B 22$ & 01 & 81 & 198.31 & 85.10 & 68982901 & 59 & 265.79 & 78.96 \\
\hline $689 B 22$ & 02 & 22 & 199.22 & 91.54 & $689 B 2901$ & 112 & 266.32 & 93.62 \\
\hline $689 B 22$ & 03 & 22 & 200.72 & 91.87 & 68982901 & 140 & 266.60 & 90.80 \\
\hline $689 B 22$ & 03 & 78 & 201.60 & 89.60 & $689 B 2902$ & 15 & 266.85 & 89.13 \\
\hline $689 B 22$ & 04 & 112 & 203.12 & 91.00 & 68982902 & 66 & 267.36 & 89.71 \\
\hline $689 B 22$ & 05 & 51 & 204.01 & 95.10 & $689 B 2902$ & 121 & 267.91 & 90.29 \\
\hline $689 B 22$ & 06 & 15 & 205.15 & 92.30 & 68982902 & 136 & 268.06 & 84.00 \\
\hline $689 \mathrm{~B} 23$ & 01 & 26 & 207.46 & 83.47 & $689 B 2903$ & 22 & 268.42 & 74.05 \\
\hline $689 B 23$ & 01 & 41 & 207.60 & 81.70 & 68982903 & 55 & 268.75 & 78.96 \\
\hline $689 \mathrm{~B} 23$ & 01 & 73 & 207.93 & 85.38 & 689B 2903 & 116 & 269.36 & 76.55 \\
\hline $689 B 23$ & 01 & 129 & 208.49 & 92.13 & $689 \mathrm{~B} 29 \mathrm{CC}$ & 22 & 269.67 & 78.21 \\
\hline 689823 & 02 & 19 & 208.89 & 92.79 & 689B 3001 & 25 & 275.15 & 85.46 \\
\hline $689 B 23$ & 02 & 73 & 209.43 & 94.04 & 689B 3001 & 76 & 275.66 & 95.54 \\
\hline $689 B 23$ & 02 & 129 & 209.99 & 90.54 & $689 B \quad 3001$ & 128 & 276.18 & 82.46 \\
\hline $689 B 23$ & 02 & 136 & 210.06 & 89.50 & 689B 3001 & 135 & 276.25 & 84.00 \\
\hline $689 B 23$ & 03 & 26 & 210.46 & 82.96 & $689 B \quad 3002$ & 15 & 276.55 & 72.38 \\
\hline $689 B 23$ & 03 & 73 & 210.93 & 91.63 & $689 B 3002$ & 75 & 277.15 & 83.96 \\
\hline $689 \mathrm{~B} 23$ & 03 & 107 & 211.27 & 93.30 & $689 B \quad 3002$ & 129 & 277.69 & 90.71 \\
\hline $689 B 23$ & 03 & 129 & 211.49 & 94.37 & $689 \mathrm{~B} 3003$ & 18 & 278.08 & 89.21 \\
\hline $689 B 23$ & 04 & 19 & 211.89 & 92.80 & $689 B 3003$ & 63 & 278.53 & 64.64 \\
\hline $689 B 23$ & 04 & 84 & 212.54 & 92.37 & $689 \mathrm{~B} 3003$ & 115 & 279.05 & 89.04 \\
\hline $689 B 23$ & $\mathrm{CC}$ & 9 & 212.76 & 91.87 & $689 \mathrm{~B} 30 \mathrm{CC}$ & 4 & 279.18 & 83.13 \\
\hline $689 B 24$ & 01 & 20 & 217.10 & 91.13 & $689 \mathrm{~B} 30 \mathrm{CC}$ & 8 & 279.22 & 95.29 \\
\hline $689 B 24$ & 01 & 63 & 217.53 & 93.04 & $689 \mathrm{~B} 32 \mathrm{CC}$ & 23 & 291.51 & 90.00 \\
\hline $689 B 24$ & 01 & 109 & 217.99 & 98.87 & & & & \\
\hline
\end{tabular}

689B 24

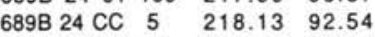

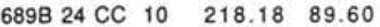

$\begin{array}{lllll}689 \mathrm{~B} & 24 \mathrm{CC} \quad 33 \quad 218.41 & 85.54\end{array}$ $\begin{array}{llllll}6898 & 25 & 01 & 7 & 226.67 & 77.88\end{array}$

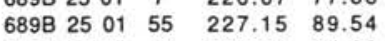
689B $2501 \quad 59 \quad 227.19 \quad 94.30$ $\begin{array}{llllll}6898 & 25 & 01 & 119 & 227.79 & 97.29\end{array}$

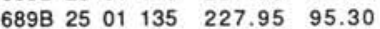

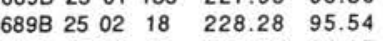
$\begin{array}{llllll}689 B & 25 & 02 & 50 & 228.60 & 94.05\end{array}$

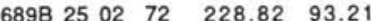
$\begin{array}{llllll}689 B & 25 & 02 & 122 & 229.32 & 91.29\end{array}$ $\begin{array}{llllll}689 B & 25 & 03 & 60 & 230.20 & 95.30\end{array}$ $\begin{array}{lllllll}689 \mathrm{~B} & 25 & 03 & 108 & 230.68 & 87.13\end{array}$ $\begin{array}{llllll}689 \mathrm{~B} & 25 & 03 & 125 & 230.85 & 92.88\end{array}$ $\begin{array}{llllll}6898 & 25 & 04 & 57 & 231.67 & 93.46\end{array}$ $\begin{array}{lllllll}689 \mathrm{~B} & 25 & 04 & 112 & 232.22 & 92.30\end{array}$

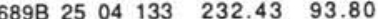

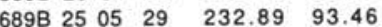
$\begin{array}{llllll}689 B & 25 & 05 & 124 & 233.84 & 93.21\end{array}$ $\begin{array}{llllll}689 B & 25 & 06 & 64 & 234.74 & 92.30\end{array}$

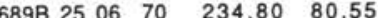
$\begin{array}{llllll}689 B & 25 & 06 & 108 & 235.18 & 91.71\end{array}$ $\begin{array}{lllll}689 \mathrm{~B} & 25 \mathrm{CC} & 5 & 235.26 & 92.88\end{array}$ $\begin{array}{lllll}689 \mathrm{~B} 25 \mathrm{CC} & 20 & 235.41 & 85.88\end{array}$

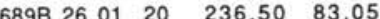
689B $2601 \quad 70 \quad 237.00 \quad 95.96$ $\begin{array}{llllll}689 B & 26 & 01 & 130 & 237.60 & 95.29\end{array}$ $\begin{array}{lllll}689 \mathrm{~B} & 26 \mathrm{CC} & 18 & 238.16 & 85.96\end{array}$

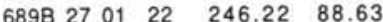

Table 2. Calcium carbonate percentages for Hole $689 \mathrm{C}$, taken between 0.20 and 27.50 mbsf depths. Data plotted in Figure 4.

\begin{tabular}{|c|c|c|c|c|}
\hline \multicolumn{3}{|c|}{$\begin{array}{l}\text { Hole, core, sec. } \\
\text { interval }(\mathrm{cm})\end{array}$} & $\begin{array}{l}\text { Depth } \\
\text { (mbsf) }\end{array}$ & $\begin{array}{c}\mathrm{CaCO} 3 \\
(\%)\end{array}$ \\
\hline $689 \mathrm{C}$ & 101 & 15 & 00.15 & 05.10 \\
\hline $689 \mathrm{C}$ & 101 & 20 & 00.20 & 05.12 \\
\hline $689 \mathrm{C}$ & 101 & 80 & 00.80 & 08.16 \\
\hline $689 \mathrm{C}$ & 101 & 135 & 01.35 & 00.50 \\
\hline $689 \mathrm{C}$ & 102 & 15 & 01.65 & 00.30 \\
\hline $689 \mathrm{C}$ & 102 & 20 & 01.70 & 01.24 \\
\hline $689 \mathrm{C}$ & 102 & 80 & 02.30 & 00.25 \\
\hline $689 \mathrm{C}$ & 102 & 135 & 02.85 & 00.75 \\
\hline $689 \mathrm{C}$ & 103 & 15 & 03.15 & 00.30 \\
\hline $689 \mathrm{C}$ & 103 & 20 & 03.20 & 02.16 \\
\hline $689 C$ & 103 & 80 & 03.80 & 00.17 \\
\hline $689 \mathrm{C}$ & 103 & 135 & 04.35 & 00.25 \\
\hline $689 \mathrm{C}$ & 104 & 15 & 04.65 & 00.20 \\
\hline $689 \mathrm{C}$ & 104 & 20 & 04.70 & 00.08 \\
\hline $689 \mathrm{C}$ & 104 & 80 & 05.30 & 01.58 \\
\hline $689 \mathrm{C}$ & 104 & 135 & 05.85 & 00.25 \\
\hline $689 \mathrm{C}$ & 105 & 15 & 06.15 & 00.30 \\
\hline $689 \mathrm{C}$ & 105 & 20 & 06.20 & 00.25 \\
\hline $689 \mathrm{C}$ & 105 & 80 & 06.80 & 00.66 \\
\hline $689 \mathrm{C}$ & 105 & 135 & 07.35 & 01.41 \\
\hline $689 \mathrm{C}$ & 106 & 15 & 07.65 & 00.30 \\
\hline $689 \mathrm{C}$ & 106 & 20 & 07.70 & 01.33 \\
\hline $689 \mathrm{C}$ & 106 & 80 & 08.30 & 03.99 \\
\hline $689 \mathrm{C}$ & 106 & 135 & 08.85 & 01.16 \\
\hline $689 \mathrm{C}$ & 107 & 15 & 09.15 & 00.30 \\
\hline $689 \mathrm{C}$ & 107 & 20 & 09.20 & 02.91 \\
\hline $689 \mathrm{C}$ & 301 & 20 & 18.30 & 21.52 \\
\hline $689 C$ & 301 & 80 & 18.90 & 06.91 \\
\hline $689 \mathrm{C}$ & 301 & 90 & 19.00 & 07.60 \\
\hline $689 \mathrm{C}$ & 301 & 135 & 19.45 & 00.42 \\
\hline $689 \mathrm{C}$ & 302 & 20 & 19.80 & 00.42 \\
\hline $689 \mathrm{C}$ & 302 & 80 & 20.40 & 00.33 \\
\hline $689 \mathrm{C}$ & 302 & 90 & 20.50 & 00.50 \\
\hline $689 \mathrm{C}$ & 302 & 135 & 20.95 & 02.42 \\
\hline $689 \mathrm{C}$ & 303 & 20 & 21.30 & 00.83 \\
\hline $689 \mathrm{C}$ & 303 & 80 & 21.90 & 00.25 \\
\hline $689 \mathrm{C}$ & 303 & 90 & 22.00 & 00.30 \\
\hline $689 \mathrm{C}$ & 303 & 135 & 22.45 & 00.17 \\
\hline $689 \mathrm{C}$ & 304 & 20 & 22.80 & 02.33 \\
\hline $689 \mathrm{C}$ & 304 & 80 & 23.40 & 45.81 \\
\hline $689 \mathrm{C}$ & 304 & 90 & 23.50 & 37.50 \\
\hline $689 \mathrm{C}$ & 304 & 135 & 23.95 & 19.57 \\
\hline $689 \mathrm{C}$ & 305 & 20 & 24.30 & 04.08 \\
\hline $689 C$ & 305 & 80 & 24.90 & 04.08 \\
\hline $689 C$ & 305 & 90 & 25.00 & 06.20 \\
\hline $689 \mathrm{C}$ & 305 & 135 & 25.45 & 00.92 \\
\hline $689 C$ & 306 & 20 & 25.80 & 03.08 \\
\hline $689 \mathrm{C}$ & 306 & 80 & 26.40 & 00.08 \\
\hline $689 \mathrm{C}$ & 306 & 90 & 26.50 & 00.30 \\
\hline $689 \mathrm{C}$ & 306 & 135 & 26.95 & 00.25 \\
\hline $689 \mathrm{C}$ & 307 & 20 & 27.30 & 00.50 \\
\hline $689 \mathrm{C}$ & 307 & 42 & 27.52 & 02.80 \\
\hline
\end{tabular}

nance continues into the Pleistocene where it is reversed by the reappearance of calcium carbonate sediments. The Pleistocene calcium carbonates, in contrast to the rest of the Cenozoic carbonates, are dominated by foraminifers rather than nannofossils.

\section{SUMMARY}

Approximately 1100 calcium carbonate measurements were made on Upper Cretaceous through Pleistocene sediments from two sites on Maud Rise, Weddell Sea. Percentage of calcium carbonate shows moderate variability in the Upper Cretaceous through upper Eocene sediments, with a slight, general decrease from the upper Paleocene to lower/middle Eocene. Variations are primarily attributed to changes in the relative abundance of terrigenous and biogenic carbonate component.

Fluctuations become more pronounced in the Oligocene, with a distinct decrease in calcium carbonate in the upper Oligocene 
Table 3. Calcium carbonate percentages for Hole 689D, taken between 18.90 and 56.20 mbsf depths. Data plotted in Figure 5.

\begin{tabular}{|c|c|c|c|c|}
\hline \multicolumn{3}{|c|}{$\begin{array}{l}\text { Hole, core, sec. } \\
\text { interval (cm) }\end{array}$} & $\begin{array}{l}\text { Depth } \\
\text { (mbsf) }\end{array}$ & $\begin{array}{c}\mathrm{CaCO} 3 \\
(\%)\end{array}$ \\
\hline $689 D$ & 101 & 80 & 18.90 & 22.27 \\
\hline 6890 & 101 & 135 & 19.45 & 12.57 \\
\hline $689 D$ & 102 & 80 & 20.40 & 00.67 \\
\hline 689D & 102 & 135 & 20.95 & 00.00 \\
\hline 689D & 103 & 20 & 21.30 & 02.41 \\
\hline $689 D$ & 103 & 80 & 21.90 & 02.63 \\
\hline 689D & 103 & 135 & 22.45 & 00.42 \\
\hline 6890 & 104 & 20 & 22.80 & 35.40 \\
\hline 689D & 104 & 80 & 23.40 & 31.74 \\
\hline $689 D$ & 104 & 135 & 23.95 & 24.40 \\
\hline $689 D$ & 105 & 80 & 24.90 & 00.49 \\
\hline $689 D$ & 106 & 20 & 25.80 & 00.42 \\
\hline 689D & 106 & 80 & 26.40 & 00.99 \\
\hline 689D & 107 & 20 & 27.30 & 00.17 \\
\hline 689D & 201 & 20 & 27.80 & 00.33 \\
\hline $689 D$ & 201 & 80 & 28.40 & 00.42 \\
\hline 689D & 201 & 135 & 28.95 & 00.99 \\
\hline $689 D$ & 202 & 20 & 29.30 & 00.17 \\
\hline $689 D$ & 202 & 80 & 29.90 & 02.74 \\
\hline 689D & 202 & 135 & 30.45 & 00.08 \\
\hline $689 D$ & 203 & 20 & 30.80 & 00.08 \\
\hline $689 D$ & 203 & 80 & 31.40 & 76.96 \\
\hline $689 \mathrm{D}$ & 203 & 135 & 31.95 & 54.06 \\
\hline $689 D$ & 204 & 20 & 32.30 & 56.06 \\
\hline 689D & 204 & 135 & 33.45 & 83.33 \\
\hline 689D & 205 & 20 & 33.80 & 84.63 \\
\hline 689D & 205 & 80 & 34.40 & 80.88 \\
\hline $689 D$ & 205 & 135 & 34.95 & 73.47 \\
\hline 689D & 206 & 20 & 35.30 & 80.88 \\
\hline 689D & 206 & 80 & 35.90 & 78.89 \\
\hline $689 D$ & 206 & 135 & 36.45 & 83.38 \\
\hline $689 D$ & 207 & 20 & 36.80 & 65.97 \\
\hline $689 D$ & 207 & 80 & 37.40 & 81.88 \\
\hline 689D & 301 & 19 & 37.49 & 81.47 \\
\hline $689 D$ & 301 & 134 & 38.64 & 92.79 \\
\hline $689 D$ & 302 & 19 & 38.99 & 93.96 \\
\hline 689D & 302 & 80 & 39.60 & 89.96 \\
\hline $689 D$ & 302 & 134 & 40.14 & 88.38 \\
\hline 6890 & 303 & 19 & 40.49 & 94.04 \\
\hline $689 D$ & 303 & 80 & 41.10 & 90.88 \\
\hline 689D & 303 & 134 & 41.64 & 81.88 \\
\hline 689D & 304 & 19 & 41.99 & 80.30 \\
\hline $689 D$ & 304 & 80 & 42.60 & 40.56 \\
\hline $689 D$ & 304 & 134 & 43.14 & 00.25 \\
\hline $689 D$ & 305 & 19 & 43.49 & 00.83 \\
\hline 689D & 305 & 80 & 44.10 & 08.57 \\
\hline 689D & 305 & 134 & 44.64 & 00.50 \\
\hline $689 D$ & 306 & 19 & 44.99 & 04.58 \\
\hline $689 D$ & 306 & 80 & 45.60 & 08.41 \\
\hline $689 D$ & 306 & 134 & 46.14 & 05.49 \\
\hline $689 D$ & 401 & 136 & 48.36 & 19.90 \\
\hline $689 D$ & 402 & 20 & 48.70 & 36.07 \\
\hline 689D & 402 & 81 & 49.31 & 33.56 \\
\hline 689D & 402 & 136 & 49.86 & 06.33 \\
\hline $689 D$ & 403 & 20 & 50.20 & 00.00 \\
\hline 689D & 403 & 81 & 50.81 & 01.66 \\
\hline $689 D$ & 403 & 137 & 51.37 & 00.25 \\
\hline 689D & 404 & 20 & 51.70 & 01.33 \\
\hline 689D & 404 & 80 & 52.30 & 00.83 \\
\hline $689 D$ & 404 & 137 & 52.87 & 57.89 \\
\hline 6890 & 405 & 20 & 53.20 & 79.97 \\
\hline $689 D$ & 405 & 80 & 53.80 & 22.24 \\
\hline 6890 & 405 & 138 & 54.38 & 44.90 \\
\hline 6890 & 406 & 20 & 54.70 & 70.47 \\
\hline $689 D$ & 406 & 80 & 55.30 & 46.81 \\
\hline $689 D$ & 406 & 139 & 55.89 & 39.40 \\
\hline $689 D$ & 407 & 20 & 56.20 & 34.90 \\
\hline
\end{tabular}

and lower middle Miocene. These variations are attributed to relative changes in the siliceous vs. carbonate component. Calcium carbonate again becomes the dominant component in the middle and lower upper Miocene, followed by almost exclusive biosiliceous sedimentation in the upper Miocene and Pliocene. Calcium carbonate sediments were deposited again in the Pleistocene.

\section{ACKNOWLEDGMENTS}

Samples were taken aboard the JOIDES Resolution and by the curatorial staff at the East Coast Repository. Caryn Smith, Hega Jensvold, and Youngtang Shan performed most of the analyses. Kahlid Mahmood and Caryn Smith entered the data into the computer and plotted the results. This manuscript was substantially improved by a review by J. P. Kennett. Funds for this study were provided by USSAC.

\section{REFERENCES}

Barker, P. F., Kennett, J. P., et al., 1988. Proc. ODP, Init. Repts., 113: College Station, TX (Ocean Drilling Program).

Berggren, W. A., Kent, D. V., Flynn, J. J., and Van Couvering, J. A., 1985a. The Neogene: Part 2 Neogene geochronology and chronostratigraphy. In Snelling, N. J. (Ed.), Geochronology of the Geological Record, Geol. Soc. London, Mem. 10:211-260. 1985 b. Paleogene geochronology and chronostratigraphy. In Snelling, N. J. (Ed.), Geochronology of the Geological Record, Geol. Soc. London, Mem. 10:141-195.

Dunn, D. A., and Moore, T. C., Jr., 1981. Late Miocene-Pliocene (Magnetic Epoch 9-Gilbert Magnetic Epoch) calcium carbonate stratigraphy of the equatorial Pacific. Geol. Soc. Am. Bull., Pt. I, 92:104107.

Dunn, D. A., Moore, T. C., Jr., and Kegwin, L. D., Jr., 1981. Atlantictype carbonate stratigraphy in the Miocene Pacific. Nature, 291: 225-227.

Gardner, J. V., 1982. High-resolution carbonate and organic-carbon stratigraphies for the late Neogene and Quaternary from the western Caribbean and eastern equatorial Pacific. In Prell, W. L., Gardner, J. V., et al., Init. Repts., DSDP, 68: Washington (U.S. Govt. Printing Office), 347-364.

Hays, J. D., Saito, T., Opdyke, N., and Burckle, L., 1969. Pliocene/ Pleistocene sediments of the equatorial Pacific: their paleomagnetic, biostratigraphic and climate record. Geol. Soc. Am. Bull., 80:1481.

Huffman, E.W.D., 1977. Performance of a New Automatic Carbon Dioxide Coulometer. Microchem. J., 22:567-573.

Pisias, N. G., and Prell, W. L., 1985. High resolution carbonate records from the hydraulic piston cored section of Site 572. In Mayer, L., Theyer, F., et al., Init. Repts., DSDP, 85: Washington (U.S. Govt. Printing Office), 711-722.

Pisias, N. G., Shackleton, N. J., and Hall, M. A., 1985. Stable isotope and calcium carbonate records from hydraulic piston cored Hole 574A: high-resolution records from the middle Miocene. In Mayer, L., Theyer, F., et al., Init. Repts., DSDP, 85: Washington (U.S. Govt. Printing Office), 735-748.

Prell, W. L., 1978. Upper Quaternary sediments of the Colombia Basin: spatial and stratigraphic variations. Geol. Soc. Am. Bull., 89:12411255.

Date of initial receipt: 13 June 1989

Date of acceptance: 15 November 1989

Ms 113B-199 
Table 4. Calcium carbonate percentages for Hole $690 \mathrm{~B}$, taken between 0.90 and 213.50 mbsf depths. Data plotted in Figure 6 .

Hole, core, sec. Depth $\mathrm{CaCO} \quad$ Hole, core, sec. Depth $\mathrm{CaCO}$ interval $(\mathrm{cm}) \quad(\mathrm{mbsf}) \quad(\%) \quad$ interval $(\mathrm{cm}) \quad(\mathrm{mbst}) \quad(\%)$

\begin{tabular}{|c|c|c|c|c|c|c|c|c|c|}
\hline $690 \mathrm{~B}$ & 101 & 90 & 00.90 & 76.60 & $690 \mathrm{~B}$ & 503 & 80 & 34.90 & 08.83 \\
\hline $690 \mathrm{~B}$ & 102 & 90 & 01.70 & 72.00 & $690 \mathrm{~B}$ & 503 & 89 & 34.99 & 36.30 \\
\hline $690 \mathrm{~B}$ & 201 & 20 & 02.30 & 26.57 & $690 \mathrm{~B}$ & 503 & 135 & 35.45 & 00.60 \\
\hline $690 \mathrm{~B}$ & 106 & 90 & 02.60 & 86.10 & $690 \mathrm{~B}$ & 504 & 20 & 35.80 & 00.42 \\
\hline $690 \mathrm{~B}$ & 201 & 80 & 02.90 & 04.41 & $690 \mathrm{~B}$ & 504 & 80 & 36.40 & 00.33 \\
\hline $690 \mathrm{~B}$ & 201 & 135 & 03.45 & 01.58 & $690 \mathrm{~B}$ & 504 & 135 & 36.95 & 00.58 \\
\hline $690 \mathrm{~B}$ & 202 & 20 & 03.80 & 01.25 & $690 \mathrm{~B}$ & 504 & 145 & 37.05 & 00.00 \\
\hline $690 \mathrm{~B}$ & 202 & 81 & 04.41 & 00.50 & $690 \mathrm{~B}$ & 505 & 20 & 37.30 & 00.42 \\
\hline $690 \mathrm{~B}$ & 202 & 90 & 04.50 & 00.00 & $690 \mathrm{~B}$ & 505 & 80 & 37.90 & 00.42 \\
\hline $690 \mathrm{~B}$ & 202 & 135 & 04.95 & 00.33 & $690 \mathrm{~B}$ & 505 & 89 & 37.99 & 00.80 \\
\hline $690 B$ & 203 & 14 & 05.24 & 00.60 & $690 \mathrm{~B}$ & 505 & 135 & 38.45 & 00.92 \\
\hline $690 \mathrm{~B}$ & 203 & 20 & 05.30 & 00.08 & $690 \mathrm{~B}$ & 506 & 20 & 38.80 & 00.33 \\
\hline $690 \mathrm{~B}$ & 203 & 135 & 06.45 & 00.33 & $690 \mathrm{~B}$ & 506 & 80 & 39.40 & 01.42 \\
\hline $690 \mathrm{~B}$ & 204 & 20 & 06.80 & 00.17 & $690 B$ & 506 & 135 & 39.95 & 00.67 \\
\hline $690 B$ & 204 & 81 & 07.41 & 00.17 & $690 \mathrm{~B}$ & 507 & 20 & 40.30 & 00.58 \\
\hline $690 \mathrm{~B}$ & 204 & 90 & 07.50 & 00.00 & $690 \mathrm{~B}$ & 601 & 20 & 41.00 & 23.91 \\
\hline $690 \mathrm{~B}$ & 204 & 135 & 07.95 & 00.33 & $690 \mathrm{~B}$ & 601 & 81 & 41.61 & 26.24 \\
\hline $690 \mathrm{~B}$ & 205 & 20 & 08.30 & 00.58 & $690 \mathrm{~B}$ & 601 & 89 & 41.69 & 32.80 \\
\hline $690 \mathrm{~B}$ & 205 & 81 & 08.91 & 00.08 & $690 \mathrm{~B}$ & 601 & 135 & 42.15 & 54.06 \\
\hline $690 \mathrm{~B}$ & 205 & 135 & 09.45 & 00.50 & $690 \mathrm{~B}$ & 602 & 20 & 42.50 & 15.08 \\
\hline $690 \mathrm{~B}$ & 206 & 20 & 09.80 & 00.17 & $690 \mathrm{~B}$ & 602 & 81 & 43.11 & 00.50 \\
\hline $690 \mathrm{~B}$ & 206 & 81 & 10.41 & 00.08 & $690 \mathrm{~B}$ & 602 & 135 & 43.65 & 53.23 \\
\hline $690 \mathrm{~B}$ & 206 & 90 & 10.50 & 00.00 & $690 \mathrm{~B}$ & 603 & 20 & 44.00 & 68.81 \\
\hline $690 \mathrm{~B}$ & 206 & 135 & 10.95 & 00.17 & $690 \mathrm{~B}$ & 603 & 81 & 44.61 & 70.31 \\
\hline $690 \mathrm{~B}$ & 207 & 21 & 11.31 & 00.08 & $690 \mathrm{~B}$ & 603 & 90 & 44.70 & 66.60 \\
\hline $690 \mathrm{~B}$ & 301 & 20 & 11.90 & 00.08 & $690 \mathrm{~B}$ & 603 & 135 & 45.15 & 79.30 \\
\hline $690 \mathrm{~B}$ & 301 & 82 & 12.52 & 00.25 & $690 \mathrm{~B}$ & 604 & 20 & 45.50 & 74.89 \\
\hline $690 \mathrm{~B}$ & 301 & 135 & 13.05 & 00.75 & $690 \mathrm{~B}$ & 604 & 81 & 46.11 & 60.23 \\
\hline $690 \mathrm{~B}$ & 302 & 20 & 13.40 & 00.08 & $690 \mathrm{~B}$ & 604 & 120 & 46.50 & 40.40 \\
\hline $690 \mathrm{~B}$ & 302 & 82 & 14.02 & 00.25 & $690 \mathrm{~B}$ & 605 & 20 & 47 & 53.06 \\
\hline $690 \mathrm{~B}$ & 302 & 90 & 14.10 & 00.00 & $690 \mathrm{~B}$ & 605 & 81 & 47.61 & 53.73 \\
\hline $690 \mathrm{~B}$ & 303 & 20 & 14.90 & 00.25 & $690 \mathrm{~B}$ & 605 & 89 & 47.90 & 53.30 \\
\hline $690 \mathrm{~B}$ & 303 & 82 & 15.52 & 00.08 & $690 \mathrm{~B}$ & 605 & 135 & 48.15 & 65.64 \\
\hline $690 \mathrm{~B}$ & 303 & 135 & 16.05 & 00.17 & $690 \mathrm{~B}$ & 606 & 20 & 48.50 & 64.89 \\
\hline $690 \mathrm{~B}$ & 304 & 12 & 16.32 & 00.30 & $690 \mathrm{~B}$ & 606 & 81 & 49.11 & 37.40 \\
\hline $690 \mathrm{~B}$ & 304 & 20 & 16.40 & 00.17 & $690 \mathrm{~B}$ & 606 & 135 & 49.65 & 71.80 \\
\hline $690 \mathrm{~B}$ & 304 & 82 & 17.02 & 00.00 & $690 \mathrm{~B}$ & 607 & 20 & 50.00 & 72.22 \\
\hline $690 \mathrm{~B}$ & 304 & 90 & 17.10 & 00.00 & $690 \mathrm{~B}$ & 701 & 20 & 50.60 & 63.64 \\
\hline $690 \mathrm{~B}$ & 304 & 119 & 17.39 & 00.08 & $690 \mathrm{~B}$ & 701 & 80 & 51.20 & 64.64 \\
\hline $690 \mathrm{~B}$ & 305 & 20 & 17.90 & 00.17 & $690 \mathrm{~B}$ & 701 & 89 & 51.30 & 69.00 \\
\hline $690 \mathrm{~B}$ & 305 & 84 & 18.54 & 00.17 & $690 \mathrm{~B}$ & 701 & 135 & 51.75 & 78.05 \\
\hline $690 \mathrm{~B}$ & 305 & 135 & 19.05 & 00.17 & $690 \mathrm{~B}$ & 702 & 20 & 52.10 & 79.05 \\
\hline $690 \mathrm{~B}$ & 306 & 20 & 19.40 & 00.33 & $690 \mathrm{~B}$ & 702 & 80 & 52.70 & 76.72 \\
\hline $690 \mathrm{~B}$ & 306 & 84 & 20.04 & 00.17 & $690 \mathrm{~B}$ & 702 & 135 & 53. & 74.72 \\
\hline $690 \mathrm{~B}$ & 306 & 90 & 20.10 & 00.00 & $690 \mathrm{~B}$ & 703 & 20 & 53.60 & 81.80 \\
\hline $690 \mathrm{~B}$ & 306 & 135 & 20.55 & 00.25 & $690 \mathrm{~B}$ & 703 & 80 & 54.20 & 83.97 \\
\hline $690 \mathrm{~B}$ & 307 & 20 & 20.90 & 00.17 & $690 \mathrm{~B}$ & 703 & 89 & 54.30 & 83.90 \\
\hline $690 \mathrm{~B}$ & 401 & 20 & 21.60 & 00.1 & $690 \mathrm{~B}$ & 703 & 135 & 54.75 & 70.97 \\
\hline $690 \mathrm{~B}$ & 401 & 80 & 22.20 & 00.42 & $690 \mathrm{~B}$ & 704 & 20 & 55.10 & 71.47 \\
\hline $690 \mathrm{~B}$ & 401 & 89 & 22.30 & 00.8 & $690 \mathrm{~B}$ & 704 & 80 & 55.70 & 82.72 \\
\hline $690 B$ & 401 & 135 & 22.75 & 00.08 & $690 \mathrm{~B}$ & 704 & 135 & 56.25 & 75.22 \\
\hline $690 \mathrm{~B}$ & 402 & 20 & 23.10 & 00.17 & $690 \mathrm{~B}$ & 705 & 20 & 56.60 & 81.30 \\
\hline $690 \mathrm{~B}$ & 402 & 80 & 23.70 & 00.17 & $690 \mathrm{~B}$ & 705 & 80 & 57.20 & 84.88 \\
\hline $690 \mathrm{~B}$ & 402 & 135 & 24.25 & 00.08 & $690 \mathrm{~B}$ & 705 & 89 & 57.30 & 88.60 \\
\hline $690 \mathrm{~B}$ & 403 & 20 & 24.60 & 20.24 & $690 \mathrm{~B}$ & 705 & 135 & 57.75 & 84.97 \\
\hline $690 \mathrm{~B}$ & 403 & 80 & 25.20 & 66.39 & $690 \mathrm{~B}$ & 706 & 20 & 58.10 & 89.46 \\
\hline $690 \mathrm{~B}$ & 403 & 89 & 25.30 & 71.90 & $690 \mathrm{~B}$ & 706 & 80 & 58.70 & 85.30 \\
\hline $690 \mathrm{~B}$ & 403 & 135 & 25.75 & 83.13 & $690 \mathrm{~B}$ & 706 & 135 & 59.25 & 87.88 \\
\hline $690 \mathrm{~B}$ & 404 & 14 & 26.04 & 80.90 & $690 \mathrm{~B}$ & 707 & 20 & 59.60 & 89.88 \\
\hline $690 \mathrm{~B}$ & 404 & 20 & 26.10 & 83.47 & $690 \mathrm{~B}$ & $7 \mathrm{CC}$ & 20 & 60.13 & 83.88 \\
\hline $690 \mathrm{~B}$ & 404 & 80 & 26.70 & 78.64 & $690 \mathrm{~B}$ & 801 & 20 & 60.30 & 81.13 \\
\hline $690 \mathrm{~B}$ & 404 & 135 & 27.25 & 87.38 & $690 \mathrm{~B}$ & 801 & 80 & 60.90 & 82.05 \\
\hline $690 \mathrm{~B}$ & 405 & 20 & 27.60 & 72.72 & $690 \mathrm{~B}$ & 801 & 89 & 61.00 & 83.10 \\
\hline $690 \mathrm{~B}$ & 405 & 80 & 28.20 & 86.97 & $690 \mathrm{~B}$ & 801 & 134 & 61.44 & 73.72 \\
\hline $690 \mathrm{~B}$ & 405 & 88 & 28.30 & 84.60 & $690 \mathrm{~B}$ & 802 & 20 & 61.80 & 84.47 \\
\hline $690 \mathrm{~B}$ & 405 & 135 & 28.75 & 85.30 & $690 \mathrm{~B}$ & 802 & 80 & 62.40 & 85.47 \\
\hline $690 \mathrm{~B}$ & 406 & 20 & 29.10 & 83.72 & $690 \mathrm{~B}$ & 802 & 134 & 62.94 & 81.47 \\
\hline $690 \mathrm{~B}$ & 406 & 80 & 29.70 & 84.22 & $690 \mathrm{~B}$ & 803 & 20 & 63.30 & 81.38 \\
\hline $690 \mathrm{~B}$ & 406 & 135 & 30.25 & 77.97 & $690 \mathrm{~B}$ & 803 & 80 & 63.90 & 47.73 \\
\hline $690 \mathrm{~B}$ & 501 & 20 & 31.30 & 83.27 & $690 \mathrm{~B}$ & 803 & 89 & 64.00 & 55.70 \\
\hline $690 \mathrm{~B}$ & 501 & 80 & 31.90 & 43.23 & $690 \mathrm{~B}$ & 803 & 134 & 64.44 & 39.23 \\
\hline $690 \mathrm{~B}$ & 501 & 88 & 31.98 & 84.30 & $690 B$ & 804 & 20 & 64.80 & 70.22 \\
\hline $690 \mathrm{~B}$ & 501 & 135 & 32.45 & 86.97 & $690 \mathrm{~B}$ & 804 & 134 & 65.94 & 73.89 \\
\hline $690 \mathrm{~B}$ & 502 & 20 & 32.80 & 85.13 & $690 \mathrm{~B}$ & 805 & 20 & 66.30 & 72.72 \\
\hline 690 & 502 & 80 & 33.40 & 85.38 & $690 \mathrm{~B}$ & 805 & 89 & 67.00 & 74.40 \\
\hline $690 \mathrm{~B}$ & 502 & 135 & 33.95 & 13.24 & $690 \mathrm{~B}$ & 806 & 20 & 67.80 & 76.14 \\
\hline $690 \mathrm{~B}$ & 503 & 20 & 34.30 & 01.00 & $690 \mathrm{~B}$ & 806 & 80 & 68.40 & 78.97 \\
\hline
\end{tabular}

Hole, core, sec. Depth $\mathrm{CaCO} 3$ interval (cm) (mbsf) (\%)

Hole, core, sec. interval $(\mathrm{cm})$

Depth $\mathrm{CaCO} 3$ (mbsf) (\%)

$\begin{array}{lllllllll}690 \mathrm{~B} & 806 \quad 134 & 68.94 \quad 77.55 & 690 \mathrm{~B} & 1204 \quad 135 \quad 104.65 \quad 85.63\end{array}$

$\begin{array}{llllll}690 \mathrm{~B} & 807 & 07 & 20 & 69.30 & 61.31\end{array}$

$\begin{array}{llllll}690 \mathrm{~B} & 9 & 01 & 20 & 70.00 & 80.47\end{array}$

$\begin{array}{llllll}690 \mathrm{~B} & 9 & 01 & 80 & 70.60 & 82.30\end{array}$

$\begin{array}{llllll}690 \mathrm{~B} & 9 & 01 & 89 & 70.70 & 76.10\end{array}$

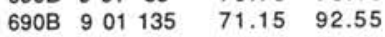

$\begin{array}{llllll}690 \mathrm{~B} & 9 & 02 & 80 & 72.10 & 67.56\end{array}$

$\begin{array}{llllll}690 \mathrm{~B} & 9 & 02 & 135 & 72.65 & 76.39\end{array}$

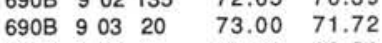

$\begin{array}{llllll}690 \mathrm{~B} & 9 & 03 & 80 & 73.60 & 63.56\end{array}$

$\begin{array}{lllllll}690 \mathrm{~B} & 9 & 03 & 90 & 73.70 & 70.50\end{array}$

$\begin{array}{llllll}690 \mathrm{~B} & 9 & 03 & 135 & 74.15 & 78.30\end{array}$

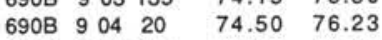

$\begin{array}{llllll}690 \mathrm{~B} & 9 & 04 & 117 & 75.57 & 72.20\end{array}$

$\begin{array}{llllll}690 \mathrm{~B} & 9 & 05 & 20 & 76.00 & 76.30\end{array}$

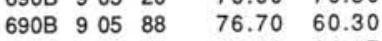

$\begin{array}{llllll}690 \mathrm{~B} & 9 & 06 & 20 & 77.50 & 64.97\end{array}$

$\begin{array}{llllll}690 \mathrm{~B} & 9 & 06 & 80 & 78.10 & 70.14\end{array}$

$\begin{array}{llllll}690 \mathrm{~B} & 9 & 06 & 135 & 78.65 & 76.05\end{array}$

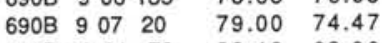

$\begin{array}{llllll}690 \mathrm{~B} & 10 & 01 & 72 & 80.12 & 63.06\end{array}$

$\begin{array}{llllll}690 \mathrm{~B} & 10 & 01 & 135 & 80.75 & 83.38\end{array}$

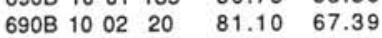

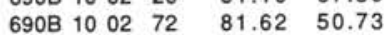

$\begin{array}{llllll}690 \mathrm{~B} & 10 & 02 & 90 & 81.80 & 61.70\end{array}$

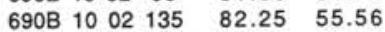

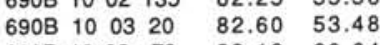

$\begin{array}{lllllll}690 \mathrm{~B} & 10 & 03 & 72 & 83.12 & 69.64\end{array}$

$\begin{array}{llllll}690 \mathrm{~B} & 10 & 03 & 135 & 83.75 & 85.30\end{array}$

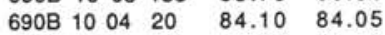

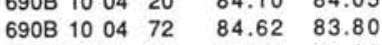

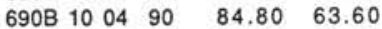

$\begin{array}{llllll}690 \mathrm{~B} & 10 & 04 & 135 & 85.25 & 63.64\end{array}$

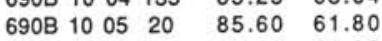

690B $10 \quad 05 \quad 72 \quad 86.12 \quad 51.06$

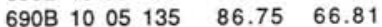

$\begin{array}{llllll}690 \mathrm{~B} & 10 & 06 & 20 & 87.10 & 65.39\end{array}$

$\begin{array}{llllll}690 \mathrm{~B} & 10 & 06 & 72 & 87.62 & 69.97\end{array}$

$\begin{array}{llllll}690 \mathrm{~B} & 10 & 06 & 90 & 87.80 & 66.00\end{array}$

$\begin{array}{llllll}690 \mathrm{~B} & 10 & 06 & 135 & 88.25 & 78.97\end{array}$

$\begin{array}{llllll}690 \mathrm{~B} & 10 & 07 & 20 & 88.60 & 75.14\end{array}$

$\begin{array}{llllll}690 \mathrm{~B} & 10 & 07 & 72 & 89.12 & 69.14\end{array}$

$\begin{array}{lllll}690 \mathrm{~B} & 1101 & 20 & 89.30 & 79.97\end{array}$

$\begin{array}{lllll}690 B & 1101 & 80 & 89.90 & 82.22\end{array}$

$\begin{array}{lllll}690 \mathrm{~B} & 1101 & 90 & 90.00 & 82.60\end{array}$

690B $1101 \quad 135 \quad 90.45 \quad 86.55$

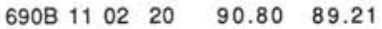

$\begin{array}{lllll}690 \mathrm{~B} 1102 & 80 & 91.40 & 81.06\end{array}$

$\begin{array}{llllll}690 \mathrm{~B} & 11 & 02 & 135 & 91.95 & 82.13\end{array}$

$\begin{array}{llllll}690 \mathrm{~B} & 11 & 03 & 20 & 92.30 & 83.38\end{array}$

$\begin{array}{llllll}690 \mathrm{~B} & 11 & 03 & 80 & 92.90 & 62.48\end{array}$

$\begin{array}{llllll}690 \mathrm{~B} & 1103 & 90 & 93.00 & 87.90\end{array}$

$\begin{array}{lllllll}690 \mathrm{~B} & 11 & 03 & 135 & 93.45 & 87.63\end{array}$

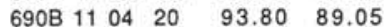

$\begin{array}{lllll}690 \mathrm{~B} 1104 & 80 & 94.40 & 91.71\end{array}$

$\begin{array}{llllll}690 B & 11 & 04 & 135 & 94.95 & 87.63\end{array}$

$\begin{array}{llllll}690 \mathrm{~B} & 11 & 05 & 20 & 95.30 & 90.71\end{array}$

$\begin{array}{llllll}690 \mathrm{~B} & 11 & 05 & 80 & 95.90 & 84.30\end{array}$

$\begin{array}{llll}690 \mathrm{~B} 1105 & 90 & 96.00 & 83.00\end{array}$

$\begin{array}{llllll}690 \mathrm{~B} & 11 & 05 & 135 & 96.45 & 89.55\end{array}$

$\begin{array}{lllll}690 B & 1106 & 20 & 96.80 & 89.05\end{array}$

$\begin{array}{lllll}690 \mathrm{~B} & 1106 & 80 & 97.40 & 94.05\end{array}$

$\begin{array}{llllll}690 B & 11 & 06 & 135 & 97.95 & 91.71\end{array}$

$\begin{array}{llllll}690 \mathrm{~B} & 11 & 07 & 20 & 98.30 & 96.38\end{array}$

$\begin{array}{llllll}690 \mathrm{~B} & 12 & 01 & 20 & 99.00 & 90.38\end{array}$

$\begin{array}{llllll}690 \mathrm{~B} & 12 & 01 & 81 & 99.61 & 91.96\end{array}$

$\begin{array}{llllll}690 B & 12 & 01 & 135 & 100.15 & 95.96\end{array}$

$\begin{array}{lllllll}690 \mathrm{~B} & 12 & 02 & 81 & 101.11 & 74.89\end{array}$

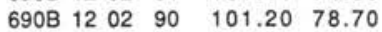

$\begin{array}{llllllllll}690 B & 12 & 02 & 135 & 101.65 & 78.14\end{array}$

$\begin{array}{lllllll}690 \mathrm{~B} & 12 & 03 & 20 & 102.00 & 78.25\end{array}$

$\begin{array}{lllllll}690 \mathrm{~B} & 12 & 03 & 81 & 102.61 & 68.56\end{array}$

$\begin{array}{lllllll}690 \mathrm{~B} & 12 & 03 & 135 & 103.15 & 74.80\end{array}$

$\begin{array}{llllll}690 \mathrm{~B} & 12 & 04 & 20 & 103.50 & 85.47\end{array}$

$\begin{array}{lllllll}690 \mathrm{~B} & 12 & 04 & 81 & 104.11 & 75.89\end{array}$

$\begin{array}{lllllll}690 \mathrm{~B} & 12 & 04 & 85 & 104.15 & 76.90\end{array}$

$\begin{array}{llllll}690 \mathrm{~B} & 12 & 04 & 120 & 104.50 & 89.70\end{array}$ $\begin{array}{llllll}690 B & 12 & 04 & 135 & 104.65 & 85.63\end{array}$

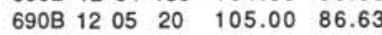

$\begin{array}{lllllll}6908 & 12 & 05 & 81 & 105.61 & 76.05\end{array}$

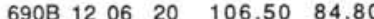

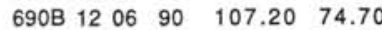

$\begin{array}{lllllll}690 B & 12 & 06 & 135 & 107.65 & 74.97\end{array}$

$\begin{array}{llllll}690 \mathrm{~B} & 13 & 01 & 19 & 108.69 & 86.54\end{array}$

$\begin{array}{llllll}690 \mathrm{~B} & 13 & 01 & 135 & 109.85 & 70.89\end{array}$

$\begin{array}{llllll}690 \mathrm{~B} & 13 & 02 & 19 & 110.19 & 83.63\end{array}$

$\begin{array}{lllllll}690 \mathrm{~B} & 13 & 02 & 81 & 110.81 & 90.38\end{array}$

$\begin{array}{llllll}690 \mathrm{~B} & 13 & 02 & 90 & 110.90 & 79.60\end{array}$ $\begin{array}{llllll}690 \mathrm{~B} & 13 & 02 & 135 & 111.35 & 79.47\end{array}$ $\begin{array}{llllll}690 \mathrm{~B} & 13 & 03 & 19 & 111.69 & 83.47\end{array}$ $\begin{array}{llllll}690 \mathrm{~B} & 13 & 03 & 135 & 112.85 & 84.55\end{array}$ $\begin{array}{llllll}690 \mathrm{~B} & 13 & 04 & 81 & 113.81 & 87.47\end{array}$

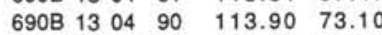
$\begin{array}{llllll}6908 & 13 & 04 & 135 & 114.35 & 78.30\end{array}$ $\begin{array}{lllllll}690 \mathrm{~B} & 13 & 05 & 19 & 114.69 & 79.47\end{array}$ $\begin{array}{llllll}690 \mathrm{~B} & 13 & 05 & 81 & 115.31 & 82.83\end{array}$ $\begin{array}{lllllll}690 \mathrm{~B} & 13 & 05 & 135 & 115.85 & 81.38\end{array}$ $\begin{array}{lllllll}690 \mathrm{~B} & 13 & 06 & 19 & 116.19 & 75.05\end{array}$ $\begin{array}{lllll}690 \mathrm{~B} & 1306 & 90 & 116.90 & 76.40\end{array}$ $\begin{array}{llllll}690 \mathrm{~B} & 13 & 06 & 135 & 117.35 & 85.49\end{array}$ $\begin{array}{lllllllllllllll}690 \mathrm{~B} & 13 & 07 & 19 & 117.69 & 80.88\end{array}$ 690B $1401 \quad 19 \quad 118.69 \quad 86.63$ $\begin{array}{llllll}690 \mathrm{~B} & 14 & 01 & 82 & 119.32 & 77.05\end{array}$ $\begin{array}{llllll}690 B & 14 & 01 & 90 & 119.40 & 78.40\end{array}$ $\begin{array}{llllll}690 \mathrm{~B} & 14 & 01 & 135 & 119.85 & 67.97\end{array}$ $\begin{array}{llllll}690 \mathrm{~B} & 14 & 02 & 19 & 120.19 & 71.47\end{array}$ $\begin{array}{llllll}690 \mathrm{~B} & 14 & 02 & 82 & 120.82 & 83.72\end{array}$ $\begin{array}{llllll}690 \mathrm{~B} & 14 & 02 & 90 & 120.90 & 86.60\end{array}$ $\begin{array}{llllll}690 \mathrm{~B} & 14 & 02 & 135 & 121.35 & 74.22\end{array}$ $\begin{array}{lllllll}690 B & 14 & 03 & 19 & 121.69 & 67.88\end{array}$

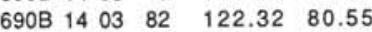
$\begin{array}{llllll}690 \mathrm{~B} & 14 & 03 & 90 & 122.40 & 81.30\end{array}$ $\begin{array}{llllll}690 \mathrm{~B} & 14 & 03 & 135 & 122.85 & 79.63\end{array}$ $\begin{array}{llllll}6908 & 14 & 04 & 19 & 123.19 & 77.47\end{array}$ $\begin{array}{lllll}690 \mathrm{~B} & 1404 & 90 & 123.60 & 81.90\end{array}$ $\begin{array}{llllll}690 \mathrm{~B} & 14 & 04 & 82 & 123.82 & 80.05\end{array}$ $\begin{array}{llllll}690 B & 14 & 04 & 135 & 124.35 & 83.72\end{array}$ 690B $14 \quad 05 \quad 19 \quad 124.69 \quad 84.92$ $\begin{array}{llllll}690 \mathrm{~B} & 14 & 05 & 90 & 125.40 & 79.30\end{array}$ $\begin{array}{llllll}690 \mathrm{~B} & 14 & 05 & 135 & 125.85 & 67.89\end{array}$ $\begin{array}{llllll}6908 & 14 & 06 & 19 & 126.19 & 73.64\end{array}$

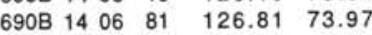
$\begin{array}{lllllll}690 \mathrm{~B} & 14 & 06 & 135 & 127.35 & 82.72\end{array}$ $\begin{array}{llllll}690 \mathrm{~B} & 14 & 07 & 19 & 127.69 & 87.88\end{array}$ $\begin{array}{llllll}690 \mathrm{~B} & 15 & 01 & 10 & 128.20 & 74.30\end{array}$

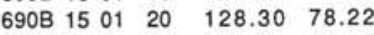
$\begin{array}{llllll}690 B & 15 & 01 & 58 & 128.68 & 81.05\end{array}$ $\begin{array}{llllll}690 B & 15 & 01 & 81 & 128.91 & 75.47\end{array}$ $\begin{array}{llllll}690 \mathrm{~B} & 15 & 01 & 108 & 129.18 & 76.22\end{array}$ $\begin{array}{lllllll}690 B & 15 & 01 & 135 & 129.45 & 77.47\end{array}$ $\begin{array}{llllll}690 \mathrm{~B} & 15 & 02 & 10 & 129.70 & 77.47\end{array}$

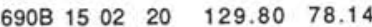

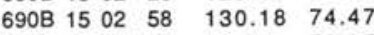
$\begin{array}{llllll}690 B & 15 & 02 & 81 & 130.41 & 78.97\end{array}$

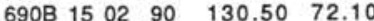
$\begin{array}{llllll}690 B & 15 & 02 & 108 & 130.68 & 64.31\end{array}$ $\begin{array}{llllll}690 B & 15 & 02 & 135 & 130.95 & 69.97\end{array}$ $\begin{array}{llllll}690 \mathrm{~B} & 15 & 03 & 10 & 131.20 & 68.64\end{array}$ $\begin{array}{llllll}690 \mathrm{~B} & 15 & 03 & 20 & 131.30 & 70.31\end{array}$

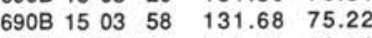
$\begin{array}{llllll}690 \mathrm{~B} & 15 & 03 & 81 & 131.91 & 68.81\end{array}$ $\begin{array}{llllll}690 \mathrm{~B} & 15 & 03 & 108 & 132.18 & 81.80\end{array}$ $\begin{array}{lllllll}690 \mathrm{~B} & 15 & 03 & 135 & 132.45 & 74.89\end{array}$ $\begin{array}{llllll}690 B & 15 & 04 & 10 & 132.70 & 71.39\end{array}$ $\begin{array}{llllll}690 \mathrm{~B} & 15 & 04 & 20 & 132.80 & 79.30\end{array}$ $\begin{array}{llllll}6908 & 15 & 04 & 58 & 133.18 & 71.89\end{array}$ $\begin{array}{lllllll}690 \mathrm{~B} & 15 & 04 & 81 & 133.41 & 74.35\end{array}$ $\begin{array}{llllll}690 B & 15 & 04 & 108 & 133.68 & 80.23\end{array}$ $\begin{array}{llllll}690 B & 15 & 04 & 135 & 133.95 & 77.72\end{array}$ $\begin{array}{llllll}690 B & 15 & 05 & 10 & 134.20 & 78.80\end{array}$

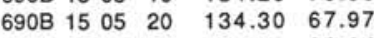
$\begin{array}{llllll}690 \mathrm{~B} & 15 & 05 & 58 & 134.68 & 66.14\end{array}$ $\begin{array}{llllll}690 \mathrm{~B} & 15 & 05 & 81 & 134.91 & 62.22\end{array}$

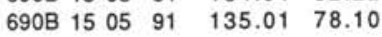


Table 4 (continued).

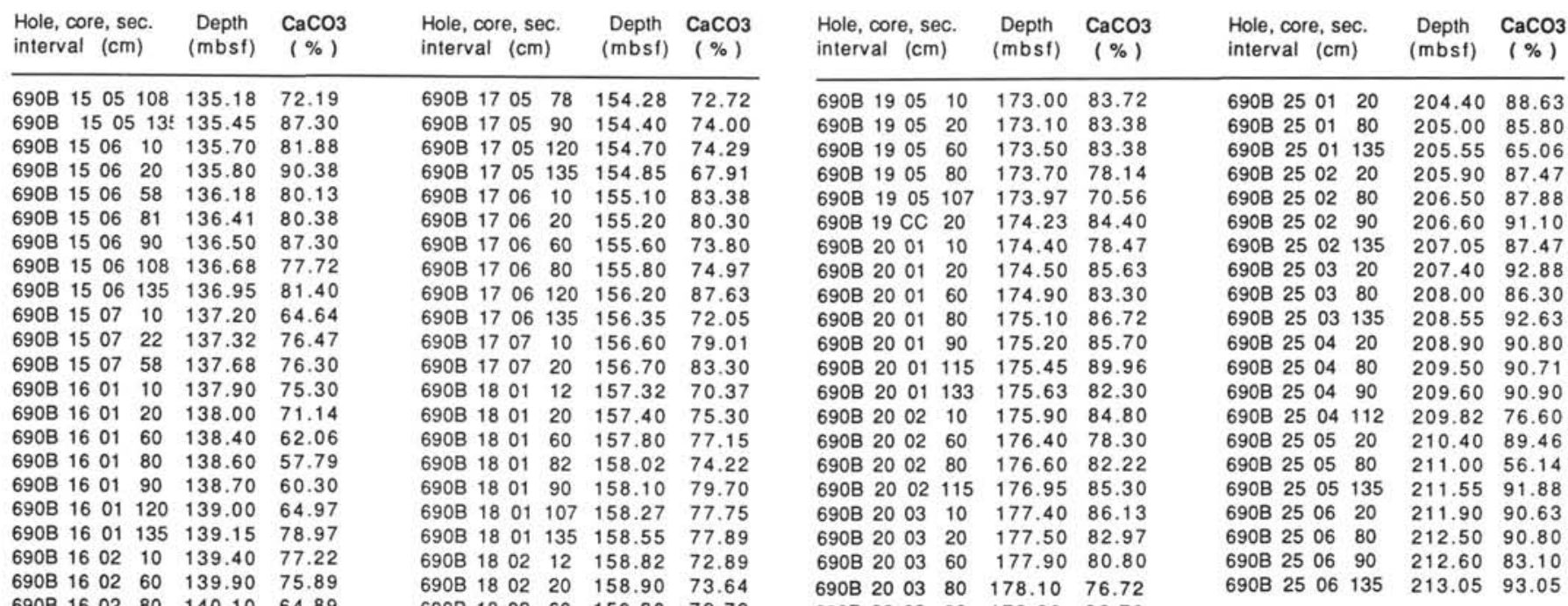

$\begin{array}{llllll}690 \mathrm{~B} & 16 & 02 & 120 & 140.50 & 71.05\end{array}$

$\begin{array}{llllll}690 \mathrm{~B} & 16 \quad 02 & 135 & 140.65 & 70.89\end{array}$

$\begin{array}{llllll}690 \mathrm{~B} & 16 & 03 & 10 & 140.90 & 71.22\end{array}$

$\begin{array}{lllllll}690 \mathrm{~B} & 16 & 03 & 20 & 141.00 & 78.30\end{array}$

$\begin{array}{llllll}690 \mathrm{~B} & 16 & 03 & 60 & 141.40 & 74.72\end{array}$

$\begin{array}{llllll}690 \mathrm{~B} & 16 & 03 & 80 & 141.60 & 89.80\end{array}$

$\begin{array}{llllll}690 \mathrm{~B} & 16 & 03 & 90 & 141.70 & 77.50\end{array}$

$\begin{array}{lllllll}690 \mathrm{~B} & 16 & 03 & 120 & 142.00 & 72.47\end{array}$

$\begin{array}{lllllll}690 \mathrm{~B} & 16 & 03 & 135 & 142.15 & 71.80\end{array}$

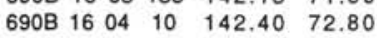

$\begin{array}{llllll}690 \mathrm{~B} & 16 & 04 & 20 & 142.50 & 58.49\end{array}$

690B $16 \quad 04 \quad 60 \quad 142.90 \quad 75.05$

$\begin{array}{llllll}690 \mathrm{~B} & 16 & 04 & 80 & 143.10 & 73.14\end{array}$

$\begin{array}{llllll}690 \mathrm{~B} & 16 & 04 & 120 & 143.20 & 73.60\end{array}$

$\begin{array}{llllll}690 \mathrm{~B} & 16 & 05 & 10 & 143.90 & 75.55\end{array}$

$\begin{array}{llllll}690 \mathrm{~B} & 16 & 05 & 20 & 144.00 & 81.22\end{array}$

$\begin{array}{lllll}690 \mathrm{~B} & 1605 & 60 & 144.40 & 75.80\end{array}$

$\begin{array}{lllllll}690 \mathrm{~B} & 16 & 05 & 80 & 144.60 & 85.22\end{array}$

$\begin{array}{lllllll}690 \mathrm{~B} & 16 & 05 & 90 & 144.70 & 83.70\end{array}$

$\begin{array}{llllll}690 \mathrm{~B} & 16 & 05 & 120 & 145.00 & 79.05\end{array}$

$\begin{array}{lllllll}690 \mathrm{~B} & 16 & 05 & 135 & 145.15 & 77.55\end{array}$

$\begin{array}{lllllll}690 \mathrm{~B} & 16 & 06 & 10 & 145.40 & 79.47\end{array}$

$\begin{array}{lllllll}690 \mathrm{~B} & 16 & 06 & 20 & 145.50 & 70.80\end{array}$

$\begin{array}{lllll}690 \mathrm{~B} & 1606 & 60 & 145.90 & 84.14\end{array}$

$\begin{array}{lllllll}690 \mathrm{~B} & 16 & 06 & 80 & 146.10 & 79.80\end{array}$

$\begin{array}{llllll}690 \mathrm{~B} & 16 & 07 & 10 & 146.90 & 65.89\end{array}$

$\begin{array}{lllllll}690 \mathrm{~B} & 16 & 07 & 20 & 147.00 & 71.05\end{array}$

690B $1701 \quad 10 \quad 147.60 \quad 78.30$

$\begin{array}{llllll}690 \mathrm{~B} & 17 & 01 & 20 & 147.70 & 66.47\end{array}$

$\begin{array}{lllll}690 \mathrm{~B} & 1701 & 60 & 148.10 & 81.01\end{array}$

$\begin{array}{lllll}690 \mathrm{~B} & 1701 & 90 & 148.10 & 81.70\end{array}$

$\begin{array}{llllll}690 \mathrm{~B} & 17 & 01 & 79 & 148.29 & 76.72\end{array}$

$\begin{array}{lllllll}690 \mathrm{~B} & 17 & 01 & 120 & 148.70 & 76.30\end{array}$

$\begin{array}{lllllll}690 \mathrm{~B} & 17 & 01 & 135 & 148.85 & 80.63\end{array}$

$\begin{array}{llllll}690 \mathrm{~B} & 17 & 02 & 10 & 149.10 & 76.73\end{array}$

690B $\begin{array}{lllll}17 & 02 & 20 & 149.20 & 79.22\end{array}$

$\begin{array}{llllll}690 \mathrm{~B} & 17 & 02 & 60 & 149.60 & 85.05\end{array}$

$\begin{array}{llllll}690 \mathrm{~B} & 17 & 02 & 79 & 149.79 & 81.06\end{array}$

$\begin{array}{llllll}690 \mathrm{~B} & 17 & 02 & 120 & 150.20 & 87.63\end{array}$

$\begin{array}{llllll}690 \mathrm{~B} & 17 & 02 & 135 & 150.35 & 86.30\end{array}$

$\begin{array}{lllllll}690 \mathrm{~B} & 17 & 03 & 10 & 150.60 & 80.59\end{array}$

$\begin{array}{lllllll}690 \mathrm{~B} & 17 & 03 & 20 & 150.70 & 78.64\end{array}$

$\begin{array}{lllllll}690 \mathrm{~B} & 17 & 03 & 60 & 151.10 & 72.80\end{array}$

$\begin{array}{llllllll}690 \mathrm{~B} & 17 & 03 & 79 & 151.29 & 73.55\end{array}$

$\begin{array}{llllll}690 \mathrm{~B} & 17 & 03 & 90 & 151.40 & 73.10\end{array}$

$\begin{array}{llllll}690 \mathrm{~B} & 17 & 03 & 120 & 151.70 & 76.30\end{array}$

$\begin{array}{lllllll}690 \mathrm{~B} & 17 & 03 & 135 & 151.85 & 76.39\end{array}$

$\begin{array}{llllll}690 \mathrm{~B} & 17 & 04 & 10 & 152.10 & 78.14\end{array}$

$\begin{array}{llllll}690 \mathrm{~B} & 1704 & 20 & 152.20 & 79.14\end{array}$

$\begin{array}{lllll}690 \mathrm{~B} & 1704 & 60 & 152.60 & 84.30\end{array}$

$\begin{array}{llllll}690 \mathrm{~B} & 17 & 04 & 80 & 152.80 & 69.14\end{array}$

$\begin{array}{llllll}690 \mathrm{~B} & 17 & 04 & 120 & 153.20 & 80.80\end{array}$

$\begin{array}{llllll}690 \mathrm{~B} & 17 & 04 & 135 & 153.35 & 74.19\end{array}$

$\begin{array}{llllll}690 \mathrm{~B} & 17 & 05 & 10 & 153.60 & 83.30\end{array}$

$\begin{array}{lllllll}690 \mathrm{~B} & 17 & 05 & 20 & 153.70 & 79.78\end{array}$

$\begin{array}{lllll}690 \mathrm{~B} & 1705 & 60 & 154.10 & 78.89\end{array}$

$\begin{array}{llllll}690 \mathrm{~B} & 18 & 02 & 60 & 159.30 & 72.72\end{array}$ $\begin{array}{llllll}690 \mathrm{~B} & 18 & 02 & 82 & 159.52 & 79.97\end{array}$ $\begin{array}{lllllll}690 \mathrm{~B} & 18 & 02 & 107 & 159.77 & 82.97\end{array}$ $\begin{array}{llllll}690 \mathrm{~B} & 18 & 02 & 135 & 160.05 & 80.55\end{array}$ $\begin{array}{lllllll}690 \mathrm{~B} & 18 & 03 & 12 & 160.32 & 74.97\end{array}$

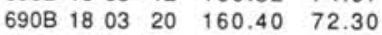
$\begin{array}{llllll}690 \mathrm{~B} & 18 & 03 & 60 & 160.80 & 81.63\end{array}$ $\begin{array}{lllllll}690 \mathrm{~B} & 18 & 03 & 82 & 161.02 & 74.88\end{array}$ $\begin{array}{lllllll}690 \mathrm{~B} & 18 & 03 & 90 & 161.10 & 79.80\end{array}$ $\begin{array}{lllllll}690 \mathrm{~B} & 18 & 03 & 107 & 161.27 & 86.05\end{array}$ $\begin{array}{llllll}690 \mathrm{~B} & 18 & 03 & 135 & 161.55 & 69.97\end{array}$ $\begin{array}{lllllll}690 \mathrm{~B} & 18 & 04 & 12 & 161.82 & 86.88\end{array}$

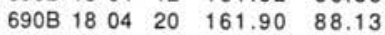
$\begin{array}{llllll}690 \mathrm{~B} & 18 & 04 & 60 & 162.30 & 91.46\end{array}$ $\begin{array}{llllll}690 \mathrm{~B} & 18 & 04 & 82 & 162.52 & 83.97\end{array}$ $\begin{array}{llllllll}690 \mathrm{~B} & 18 & 04 & 107 & 162.77 & 81.63\end{array}$ $\begin{array}{llllll}690 \mathrm{~B} & 18 & 04 & 135 & 163.05 & 80.47\end{array}$ $\begin{array}{llllll}690 \mathrm{~B} & 18 & 05 & 12 & 163.32 & 87.71\end{array}$ $\begin{array}{llllll}690 \mathrm{~B} & 18 & 05 & 20 & 163.40 & 84.05\end{array}$ $\begin{array}{lllll}690 \mathrm{~B} & 1805 & 60 & 163.80 & 80.55\end{array}$ $\begin{array}{llllll}690 \mathrm{~B} & 18 & 05 & 82 & 164.02 & 85.05\end{array}$ $\begin{array}{lllllll}690 \mathrm{~B} & 18 & 05 & 90 & 164.10 & 85.50\end{array}$ $\begin{array}{lllllll}690 \mathrm{~B} & 18 & 05 & 107 & 164.27 & 78.72\end{array}$ $\begin{array}{llllll}690 \mathrm{~B} & 18 & 05 & 135 & 164.55 & 88.05\end{array}$ $\begin{array}{llllll}690 \mathrm{~B} & 18 & 06 & 12 & 164.82 & 74.14\end{array}$ $\begin{array}{llllll}690 \mathrm{~B} & 18 & 06 & 20 & 164.90 & 78.80\end{array}$ $\begin{array}{llllll}690 \mathrm{~B} & 18 & 06 & 60 & 165.30 & 82.30\end{array}$ $\begin{array}{llllll}690 \mathrm{~B} & 18 & 06 & 82 & 165.52 & 77.86\end{array}$ $\begin{array}{lllllll}690 \mathrm{~B} & 18 & 06 & 107 & 165.77 & 77.47\end{array}$ 690B $\begin{array}{llllll}18 & 06 & 135 & 166.05 & 87.05\end{array}$ $\begin{array}{lllll}690 \mathrm{~B} & 18 \quad 07 & 12 & 166.32 & 83.80\end{array}$

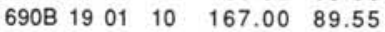
$\begin{array}{llllll}690 \mathrm{~B} & 1901 & 20 & 167.10 & 89.71\end{array}$ $\begin{array}{llllll}690 \mathrm{~B} & 19 & 01 & 60 & 167.50 & 91.55\end{array}$ $\begin{array}{llllll}690 \mathrm{~B} & 19 & 01 & 80 & 167.70 & 91.38\end{array}$ $\begin{array}{llllll}690 \mathrm{~B} & 19 & 01 & 90 & 167.80 & 94.50\end{array}$ $\begin{array}{lllllll}690 \mathrm{~B} & 19 & 01 & 107 & 167.97 & 98.54\end{array}$ $\begin{array}{llllll}690 \mathrm{~B} & 19 & 01 & 135 & 168.25 & 95.38\end{array}$ $\begin{array}{llllll}690 \mathrm{~B} & 19 & 02 & 10 & 168.50 & 89.63\end{array}$ $\begin{array}{llllll}690 \mathrm{~B} & 19 & 02 & 20 & 168.60 & 94.38\end{array}$ $\begin{array}{llllll}690 \mathrm{~B} & 19 & 02 & 60 & 169.00 & 93.96\end{array}$ $\begin{array}{llllll}690 \mathrm{~B} & 19 & 02 & 80 & 169.20 & 83.13\end{array}$ $\begin{array}{lllllll}690 \mathrm{~B} & 19 & 02 & 107 & 169.47 & 82.30\end{array}$ $\begin{array}{lllllll}690 \mathrm{~B} & 19 & 02 & 135 & 169.75 & 84.13\end{array}$ $\begin{array}{llllll}690 \mathrm{~B} & 19 & 03 & 10 & 170.00 & 68.81\end{array}$ $\begin{array}{lllllll}690 \mathrm{~B} & 19 & 03 & 20 & 170.10 & 73.97\end{array}$ $\begin{array}{lllllll}690 \mathrm{~B} & 19 & 03 & 60 & 170.50 & 67.31\end{array}$ $\begin{array}{lllllll}690 \mathrm{~B} & 19 & 03 & 80 & 170.70 & 75.64\end{array}$ $\begin{array}{llllll}690 \mathrm{~B} & 1903 & 90 & 170.80 & 66.10\end{array}$ $\begin{array}{lllllll}690 \mathrm{~B} & 19 & 03 & 107 & 170.97 & 82.55\end{array}$ $\begin{array}{lllllll}690 \mathrm{~B} & 19 & 03 & 135 & 171.25 & 82.55\end{array}$

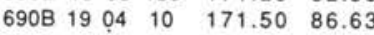
$\begin{array}{lllll}690 \mathrm{~B} & 1904 & 20 & 171.60 & 91.46\end{array}$ $\begin{array}{llllll}690 \mathrm{~B} & 19 & 04 & 60 & 172.00 & 88.21\end{array}$ $\begin{array}{llllll}690 \mathrm{~B} & 19 & 04 & 80 & 172.20 & 86.88\end{array}$ $\begin{array}{lllllll}690 \mathrm{~B} & 19 & 04 & 112 & 172.22 & 80.80\end{array}$ $\begin{array}{llllll}690 \mathrm{~B} & 19 & 04 & 107 & 172.47 & 73.39\end{array}$

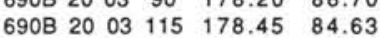
$\begin{array}{llllll}690 \mathrm{~B} & 20 & 03 & 133 & 178.63 & 80.72\end{array}$

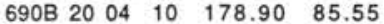
$\begin{array}{llllll}690 \mathrm{~B} & 20 & 04 & 60 & 179.40 & 78.39\end{array}$ $\begin{array}{lllll}690 \mathrm{~B} 20 & 04 & 80 & 179.60 & 79.38\end{array}$ 690B $2004 \quad 115 \quad 179.95 \quad 81.30$ $\begin{array}{llllll}690 B & 20 & 04 & 124 & 180.04 & 79.97\end{array}$

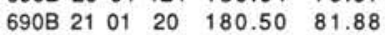

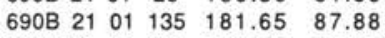
$\begin{array}{lllllll}690 \mathrm{~B} & 21 & 02 & 20 & 182.00 & 74.80\end{array}$ $\begin{array}{llllll}690 \mathrm{~B} & 21 & 02 & 90 & 182.70 & 83.70\end{array}$ $\begin{array}{llllll}690 \mathrm{~B} & 21 & 02 & 135 & 183.15 & 87.88\end{array}$ $\begin{array}{llllll}690 \mathrm{~B} & 21 & 03 & 20 & 183.50 & 88.80\end{array}$ $\begin{array}{lllllll}690 \mathrm{~B} & 21 & 03 & 71 & 184.01 & 87.30\end{array}$ $\begin{array}{llllll}690 \mathrm{~B} & 21 & 04 & 10 & 184.40 & 84.60\end{array}$ $\begin{array}{llllll}690 \mathrm{~B} & 21 & 03 & 135 & 184.65 & 82.47\end{array}$ $\begin{array}{llllll}690 \mathrm{~B} & 22 & 01 & 20 & 185.40 & 78.72\end{array}$ 690B $22 \quad 01 \quad 135 \quad 186.55 \quad 87.96$

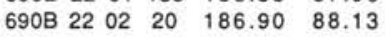
690B $22 \quad 02 \quad 90 \quad 187.30 \quad 85.80$ $\begin{array}{llllll}690 \mathrm{~B} & 22 & 02 & 80 & 187.50 & 89.13\end{array}$

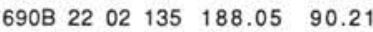
$\begin{array}{llllll}690 \mathrm{~B} & 22 & 03 & 20 & 188.40 & 90.80\end{array}$ $\begin{array}{llllll}690 \mathrm{~B} & 22 & 03 & 80 & 189.00 & 85.97\end{array}$ 690B $22 \quad 03 \quad 120 \quad 189.40 \quad 81.10$ $\begin{array}{llllll}690 \mathrm{~B} & 22 & 04 & 20 & 189.90 & 74.39\end{array}$ 690B $2204 \quad 80 \quad 190.50 \quad 82.13$

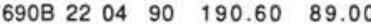

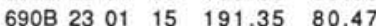

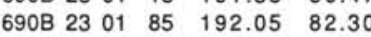
690B $2301 \quad 135 \quad 192.55 \quad 90.05$ $\begin{array}{llllll}690 \mathrm{~B} & 23 & 02 & 15 & 192.85 & 92.63\end{array}$

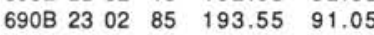
690B $23 \quad 02 \quad 90 \quad 193.60 \quad 92.70$ $\begin{array}{llllll}690 \mathrm{~B} & 23 & 02 & 135 & 194.05 & 90.55\end{array}$ $\begin{array}{llllll}690 \mathrm{~B} & 23 & 03 & 15 & 194.35 & 90.38\end{array}$ $\begin{array}{llllll}690 \mathrm{~B} & 23 & 03 & 85 & 195.05 & 88.13\end{array}$ $\begin{array}{llllll}690 \mathrm{~B} & 23 & 03 & 135 & 195.55 & 85.88\end{array}$ $\begin{array}{llllll}690 \mathrm{~B} & 23 & 04 & 15 & 195.85 & 88.30\end{array}$

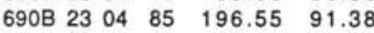

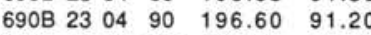
$\begin{array}{llllll}690 \mathrm{~B} & 23 & 04 & 135 & 197.05 & 85.13\end{array}$

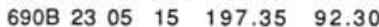

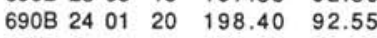
690B $2401 \quad 72 \quad 198.92 \quad 89.63$ 690B $2401135 \quad 199.55 \quad 87.96$ 690B $24 \quad 02 \quad 20 \quad 199.90 \quad 83.83$ $\begin{array}{llll}690 \mathrm{~B} & 2402 \quad 92 \quad 200.30 \quad 86.40\end{array}$ $\begin{array}{llllll}690 B & 24 & 02 & 72 & 200.42 & 86.63\end{array}$ 690B $24 \quad 02135 \quad 201.05 \quad 89.13$ $\begin{array}{llllll}690 \mathrm{~B} & 24 & 03 & 20 & 201.40 & 88.46\end{array}$ $\begin{array}{llllll}690 \mathrm{~B} & 24 & 03 & 72 & 201.92 & 87.80\end{array}$ $\begin{array}{llllll}690 \mathrm{~B} & 24 & 03 & 135 & 202.55 & 88.30\end{array}$ 690B $24 \quad 04 \quad 20 \quad 202.90 \quad 87.05$ $\begin{array}{llllll}690 \mathrm{~B} & 24 & 04 & 72 & 203.42 & 89.55\end{array}$

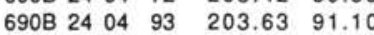


Table 5. Calcium carbonate percentages for Hole $690 \mathrm{C}$, taken between 204.30 and 294.45 mbsf depths. Data plotted in Figure 7.

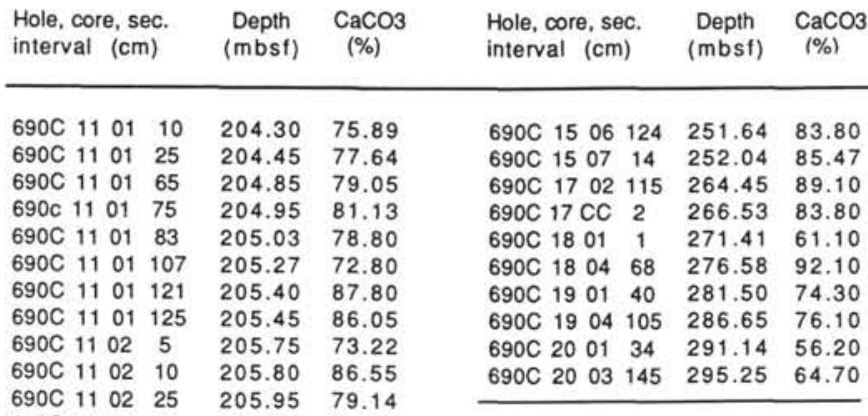

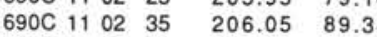

$\begin{array}{lllll}690 \mathrm{C} & 1102 & 63 & 206.33 & 89.38\end{array}$

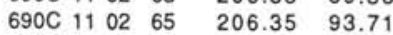

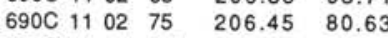

$\begin{array}{llllll}690 C & 11 & 02 & 107 & 206.77 & 89.29\end{array}$

690C $1102 \quad 111 \quad 206.81 \quad 87.81$

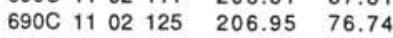

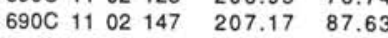

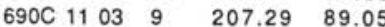

$690 \mathrm{C} \quad \begin{array}{llll}1103 & 10 & 207.30 & 85.88\end{array}$

$\begin{array}{lllll}690 \mathrm{C} & 1103 & 25 & 207.45 & 83.47\end{array}$

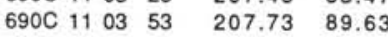

$\begin{array}{lllll}690 \mathrm{C} & 1103 & 65 & 207.85 & 88.05\end{array}$

$\begin{array}{llllll}690 \mathrm{C} & 11 & 03 & 75 & 207.95 & 84.80\end{array}$

$\begin{array}{llllll}690 \mathrm{C} & 11 & 03 & 107 & 208.27 & 92.63\end{array}$

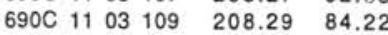

$690 \mathrm{C} \quad 1103 \quad 110 \quad 208.30 \quad 77.14$

$\begin{array}{lllll}690 \mathrm{C} & 1103 & 112 & 208.32 & 71.50\end{array}$

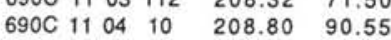

$\begin{array}{lllll}690 \mathrm{C} & 1104 & 25 & 208.95 & 85.38\end{array}$

$690 C \quad 1104 \quad 51 \quad 209.21 \quad 96.63$

$690 \mathrm{C} \quad 1104 \quad 58 \quad 209.28 \quad 91.63$

$\begin{array}{lllll}690 C & 1104 & 61 & 209.31 & 93.80\end{array}$

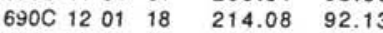

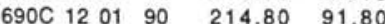

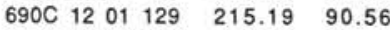

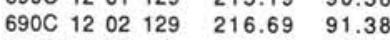

$\begin{array}{llllll}690 \mathrm{C} & 12 & 03 & 19 & 217.09 & 92.30\end{array}$

$\begin{array}{lllll}690 \mathrm{C} & 12 \mathrm{CC} & 16 & 217.64 & 97.38\end{array}$

$\begin{array}{lllll}690 \mathrm{C} & 1301 & 20 & 223.80 & 95.46\end{array}$

$\begin{array}{lllll}690 \mathrm{C} & 1301 & 80 & 224.40 & 76.64\end{array}$

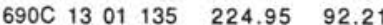

$\begin{array}{lllll}690 \mathrm{C} & 1302 & 20 & 225.30 & 98.74\end{array}$

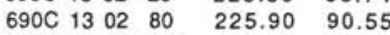

$\begin{array}{llllll}690 \mathrm{C} & 13 & 02 & 135 & 226.45 & 70.56\end{array}$

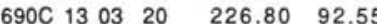

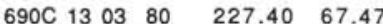

$\begin{array}{llllll}690 \mathrm{C} & 13 & 03 & 135 & 227.95 & 87.88\end{array}$

$\begin{array}{lllll}690 \mathrm{C} & 1304 & 20 & 228.30 & 89.46\end{array}$

$\begin{array}{lllll}690 \mathrm{C} & 1304 & 80 & 228.90 & 92.71\end{array}$

$\begin{array}{llllll}690 \mathrm{C} & 13 & 04 & 135 & 229.45 & 91.55\end{array}$

$\begin{array}{lllll}690 \mathrm{C} & 1305 & 20 & 229.80 & 77.55\end{array}$

$\begin{array}{lllll}690 \mathrm{C} & 1305 & 80 & 230.40 & 90.88\end{array}$

$\begin{array}{llllll}690 \mathrm{C} & 13 & 05 & 135 & 230.95 & 89.71\end{array}$

$\begin{array}{llllll}690 \mathrm{C} & 13 & 05 & 147 & 231.07 & 83.20\end{array}$

$\begin{array}{lllll}690 \mathrm{C} & 1306 & 20 & 231.30 & 87.05\end{array}$

$\begin{array}{lllll}690 \mathrm{C} & 1306 & 80 & 231.90 & 87.88\end{array}$

$\begin{array}{llllll}690 \mathrm{C} & 14 & 01 & 81 & 234.01 & 86.97\end{array}$

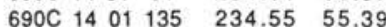

$\begin{array}{lllll}690 \mathrm{C} & 1402 & 20 & 234.90 & 88.21\end{array}$

$\begin{array}{llllll}690 \mathrm{C} & 14 & 02 & 81 & 235.51 & 77.22\end{array}$

690C $1402 \quad 135 \quad 236.05 \quad 100.00$

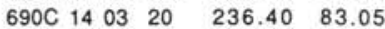

$\begin{array}{llllll}690 \mathrm{C} & 14 & 03 & 81 & 237.01 & 85.88\end{array}$

$\begin{array}{lllllll}690 \mathrm{C} & 14 & 03 & 135 & 237.55 & 84.63\end{array}$

$\begin{array}{llllll}690 \mathrm{C} & 14 & 04 & 115 & 238.95 & 88.70\end{array}$

$\begin{array}{llllll}690 \mathrm{C} & 1501 & 23 & 243.13 & 89.13\end{array}$

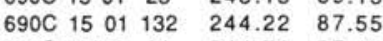

$\begin{array}{llllll}690 \mathrm{C} & 15 & 02 & 31 & 244.71 & 87.05\end{array}$

690C $15 \quad 02 \quad 135 \quad 245.75 \quad 86.80$

$\begin{array}{llllll}690 \mathrm{C} & 15 & 03 & 27 & 246.17 & 85.22\end{array}$

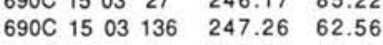

$\begin{array}{llllll}690 C & 15 & 04 & 14 & 247.54 & 48.40\end{array}$

$\begin{array}{llllll}690 \mathrm{C} & 15 & 04 & 104 & 248.44 & 89.30\end{array}$

$\begin{array}{llllll}690 \mathrm{C} & 15 & 05 & 17 & 249.07 & 80.97\end{array}$

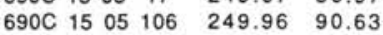

$\begin{array}{lllll}690 \mathrm{C} & 1506 & 26 & 250.66 & 77.97\end{array}$ 

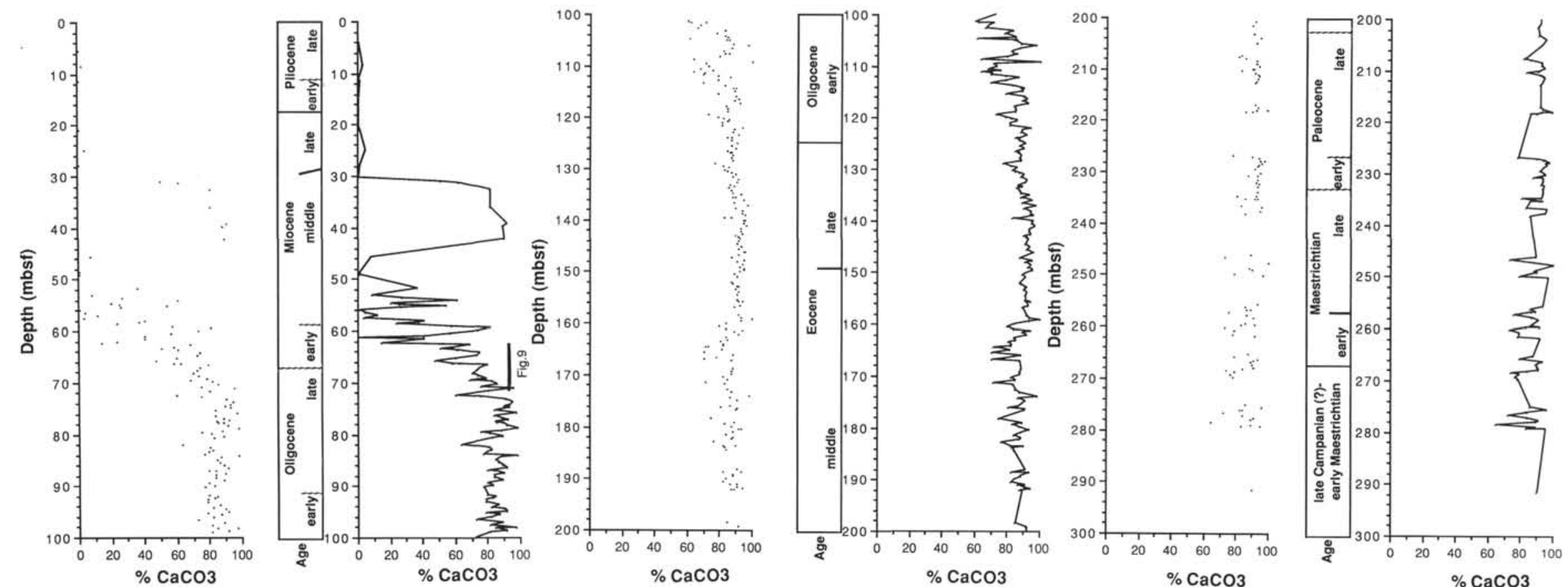

Figure 3. Calcium carbonate percentages for Hole 689B, taken between depths 4.00 and 291.50 mbsf. Ages are from Gersonde et al. (this volume) and Thomas et al. (this volume); wavy lines indicate hiatuses. Data given in Table 1 . 

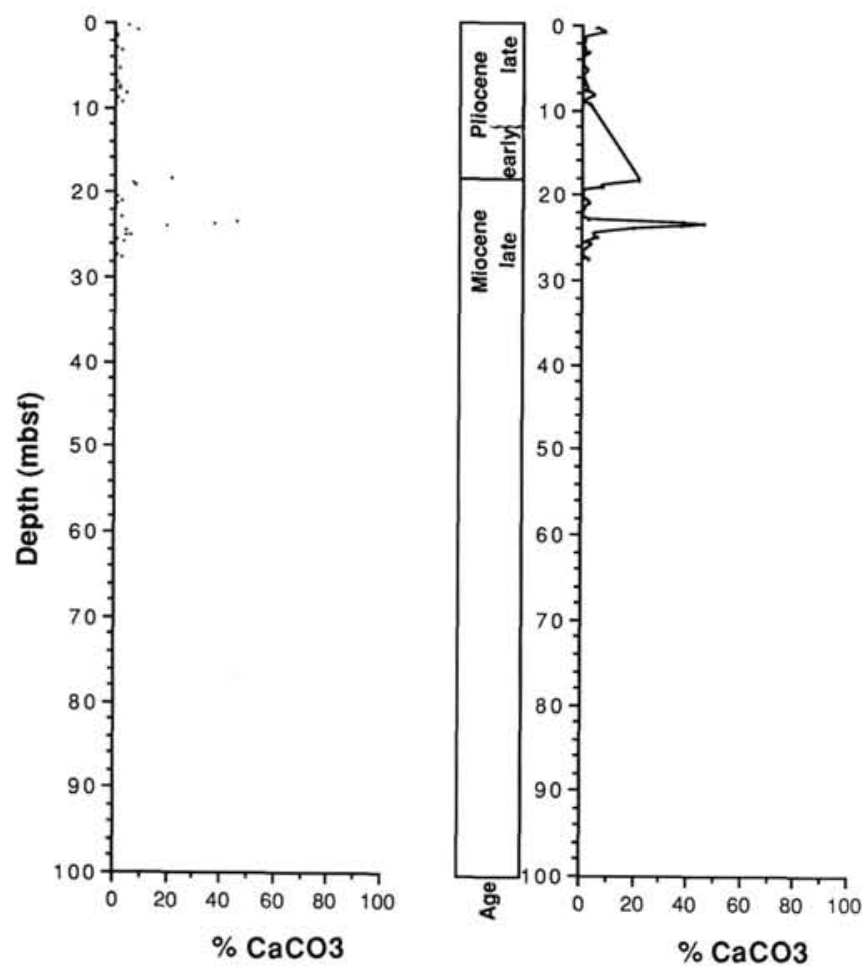

Figure 4. Calcium carbonate percentages for Hole 689C, taken between depths 0.20 and 27.50 mbsf. Ages are from Gersonde et al. (this volume) and Thomas et al. (this volume); wavy lines indicate hiatuses. Data given in Table 2 .
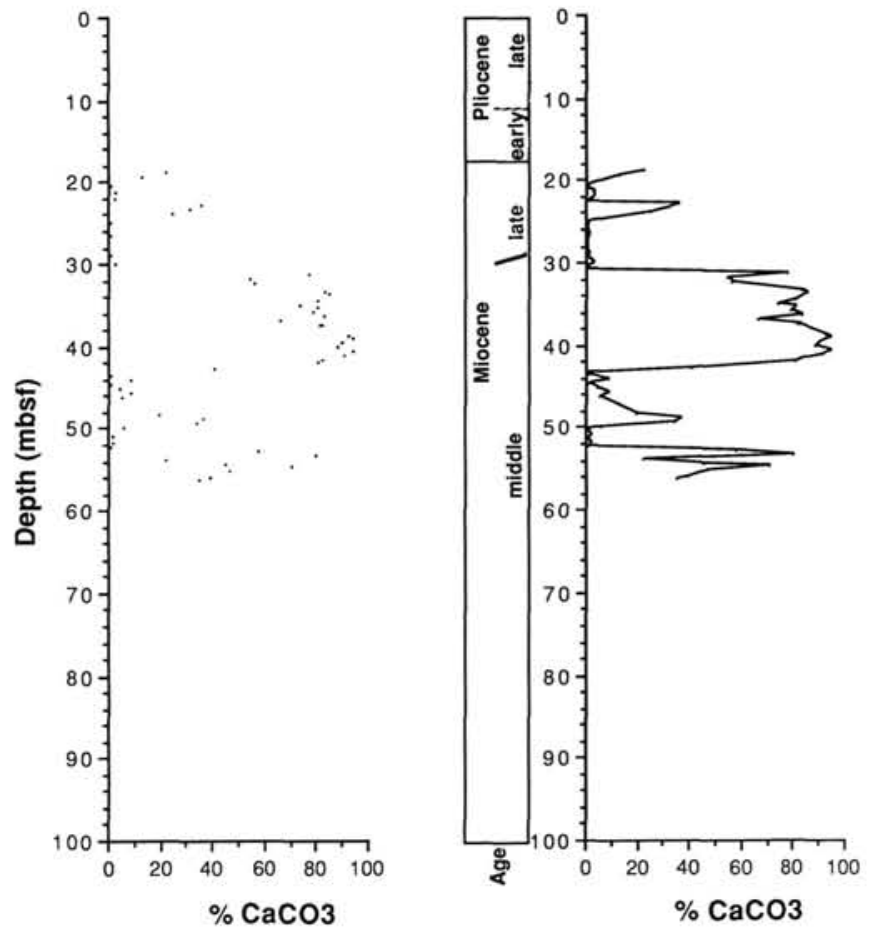

Figure 5. Calcium carbonate percentages for Hole 689D, taken between depths 18.90 and 56.20 mbsf. Ages are from Gersonde et al. (this volume) and Thomas et al. (this volume); wavy lines indicate hiatuses. Data given in Table 3. 

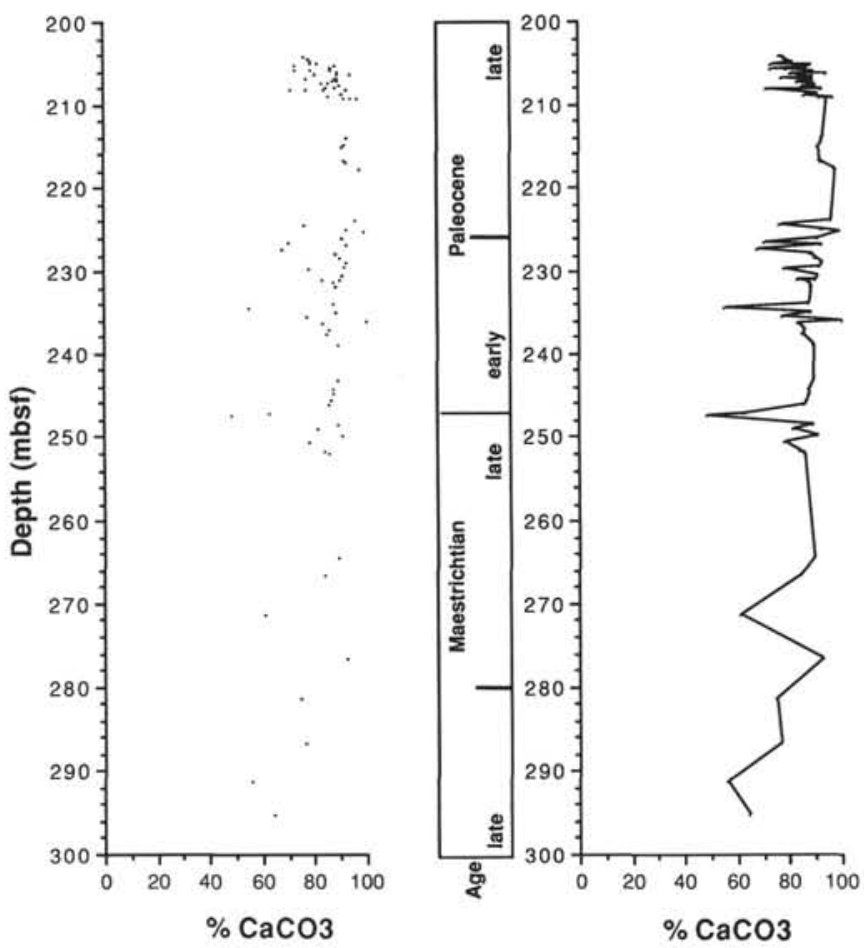

Figure 7. Calcium carbonate percentages for Hole $690 \mathrm{C}$, taken between depths 204.30 and 294.45 mbsf. Ages are from Gersonde et al. (this volume) and Thomas et al. (this volume). Data given in Table 5.

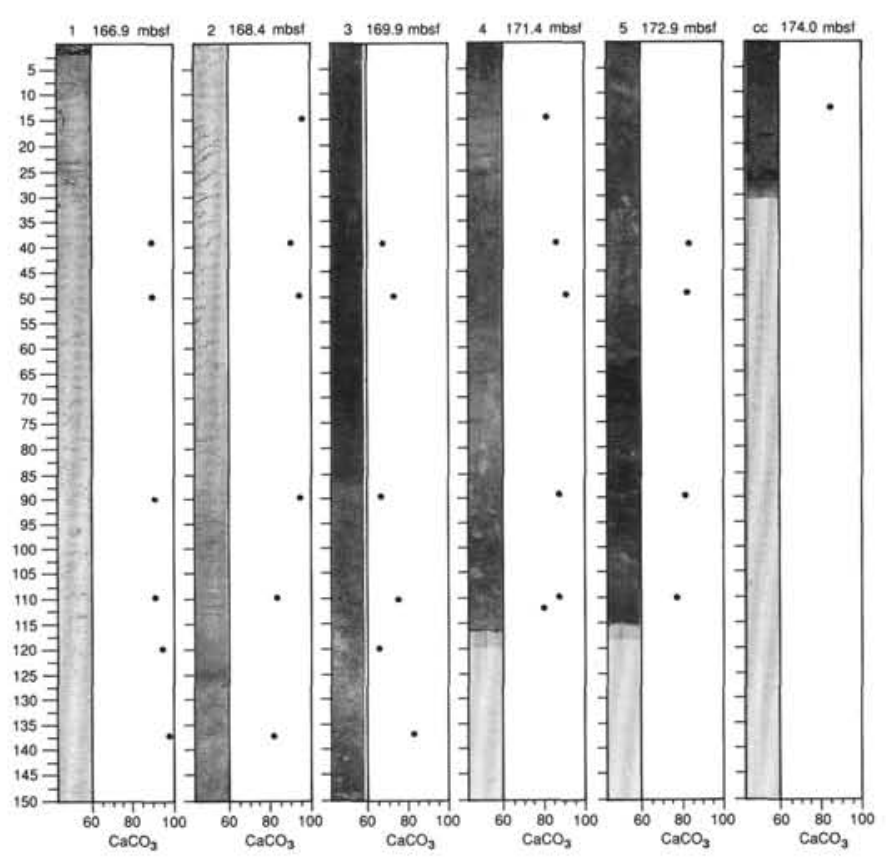

Figure 8. Calcium carbonate percentages plotted adjacent to a photograph of Core 113B-690B-19H (166.9-174.3 mbsf). Color changes are primarily due to relative changes in the clay content. Depth (mbsf) is given for the top of each section. 


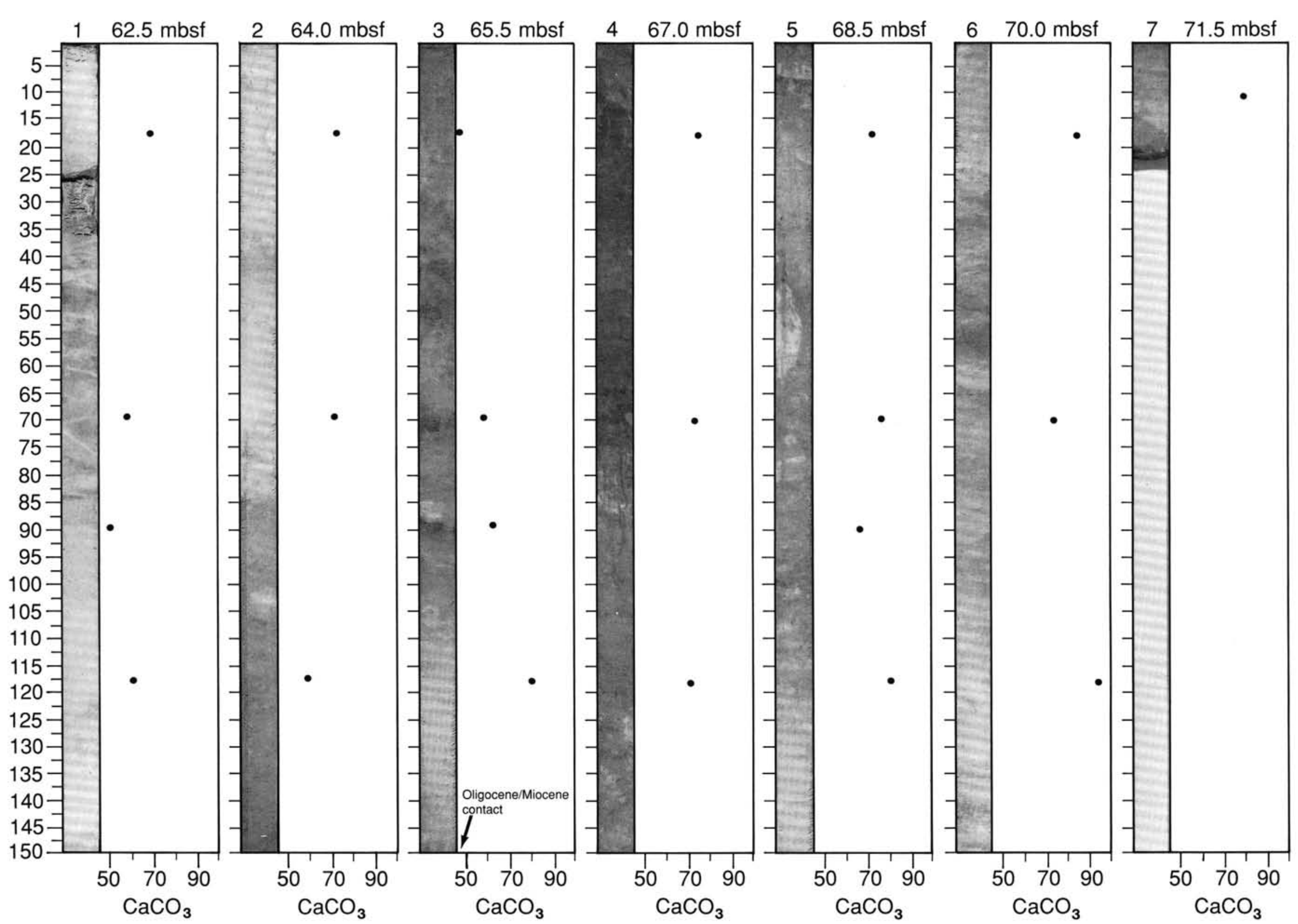

Figure 9. Calcium carbonate percentages plotted adjacent to a photograph of Core 113B-689B-8H (62.5-72.1 mbsf). Color changes are primarily due to relative changes in the siliceous vs. carbonate biogenic content. Textural changes, not readily visible in the photograph, are present. Depth (mbsf) is given for the top of each section. 